\title{
A systematic review of enhanced (or engineered) geothermal systems: past, present and future
}

\author{
Katrin Breede*, Khatia Dzebisashvili, Xiaolei Liu and Gioia Falcone
}

\author{
* Correspondence: katrin.breede@ \\ tu-clausthal.de \\ Institute of Petroleum Engineering, \\ Clausthal University of Technology, \\ Agricolastraße 10, \\ Clausthal-Zellerfeld 38678, Germany
}

\begin{abstract}
Enhanced (or engineered) geothermal systems (EGS) have evolved from the hot dry rock concept, implemented for the first time at Fenton Hill in 1977. This paper systematically reviews all of the EGS projects worldwide, based on the information available in the public domain. The projects are classified by country, reservoir type, depth, reservoir temperature, stimulation methods, associated seismicity, plant capacity and current status. Thirty five years on from the first EGS implementation, the geothermal community can benefit from the lessons learnt and take a more objective approach to the pros and cons of 'conventional' EGS systems.
\end{abstract}

Keywords: Enhanced geothermal system, Engineered geothermal system, Hot dry rock, Conventional EGS, EGS database worldwide

\section{Review}

The currently used term 'enhanced or engineered geothermal system' (EGS) has its roots in the early 1970s when a team from Los Alamos National Laboratories began the hot dry rock (HDR) project at Fenton Hill (Cummings and Morris 1979; Tester et al. 1989; Brown 1997; Duchane 1998). The concept is described in Potter et al. (1974). HDR was also known as hot fractured rock because of either the need to fracture the virtually impermeable formations or the presence of natural fractures in the hot reservoir (Wyborn et al. 2005; Goldstein et al. 2011) or as hot wet rock (HWR) when it was established that the formations were not completely dry but contained some fluids. The European EGS project at Soultz-sous-Forêts in France is an example of a HWR reservoir (Duchane 1998). Further nomenclature encountered in the literature include stimulated geothermal system, deep heat mining (Häring and Hopkirk 2002; Häring 2007) and deep earth geothermal. All of the above usually imply the use of petrothermal systems (Ilyasov et al. 2010; Gebo NDS 2012a).

Schulte et al. (2010) defined the typical geological settings for EGS, varying from igneous (e.g. Iceland), metamorphic (e.g. Lardarello, Italy), magmatic (e.g. Soultz, France) and sedimentary (e.g. Groß Schönebeck and Horstberg, Germany).

According to Potter et al. (1974), the most suitable rock type for HDR is granite or other crystalline basement rock; temperatures should vary from $150^{\circ} \mathrm{C}$ to $500^{\circ} \mathrm{C}$ at depths in the order of 5 to $6 \mathrm{~km}$, with an average flow rate over a 10-year reservoir lifetime of $265 \mathrm{l} / \mathrm{s}$, with hydraulic fracturing achieving a contact surface area of approximately $16 \mathrm{~km}^{2}$, an average thermal capacity of $250 \mathrm{MW}_{\text {th }}$ that could be obtained from the surface heat exchanger, and with pressurized water entering at $280^{\circ} \mathrm{C}$ and 
leaving at $65^{\circ} \mathrm{C}$. Based on these criteria, the potential electrical power that could be generated might amount to $50 \mathrm{MW}_{\mathrm{e}}$ at a net efficiency of $20 \%$.

Over the years, different definitions of EGS have been proposed, covering a broad variety of rock types, depth, temperature, reservoir permeability and porosity, type of stimulation technique involved, etc. Below are four examples of recent EGS definitions in the public domain.

1. The Massachusetts Institute of Technology (MIT) led an interdisciplinary panel which defined EGS as 'engineered reservoirs that have been created to extract economical amounts of heat from low permeability and/or porosity geothermal resources. For this assessment, this definition has been adapted to include all geothermal resources that are currently not in commercial production and require stimulation or enhancement. EGS would exclude high-grade hydrothermal but include conduction dominated, low permeability resources in sedimentary and basement formations, as well as geopressured, magma and low grade, unproductive hydrothermal resources. Co-produced hot water from oil and gas production is included as an unconventional EGS resource type that could be developed in the short term and possibly provide a first step to more classical EGS exploitation' (MIT et al. 2006a).

2. The Australian Geothermal Reporting Code Committee considered EGS as 'a body of rock containing useful energy, the recoverability of which has been increased by artificial means such as fracturing' (AGRCC 2010).

3. Williams et al. (2011) proposed that 'EGS comprise the portion of a geothermal resource for which a measureable increase in production over its natural state is or can be attained through mechanical, thermal, and/or chemical stimulation of the reservoir rock. In this definition, there are no restrictions on temperature, rock type or pre-existing geothermal exploitation'.

4. The BMU (2011) defines enhanced geothermal systems as creating or enhancing a heat exchanger in deep and low permeable hot rocks using stimulation methods. Following BMU's definition, EGS embraces not only HDR but also deep heat mining, hot wet rock, hot fractured rock, stimulated geothermal systems, and stimulated hydrothermal systems.

Clearly, the geothermal community lacks a universal definition of EGS, which may simply be taken as 'unconventional geothermal systems', diverging significantly from the initial HDR concept. This lack of clarity may constitute a potential obstacle to the implementation of tailored subsidy programmes.

In this study, the MIT definition is adopted with the only difference that geopressured and magmatic systems and also co-produced hot water from hydrocarbon wells are excluded. The reasons for this particular choice are that the MIT report was (and still is) regarded as a milestone report towards the development of EGS; also, from an engineering point of view, it is perhaps one of the most comprehensive definitions. On the other hand, it does not enter into the details of the different stimulation approaches and associated consequences for different EGS systems. Recently, for example, Jung (2013) has reconstructed the background to contemporary EGS: from the original HDR concept based on multi-zone hydraulic fracturing in 
competent crystalline formations, through that of open-hole massive injection in naturally fractured crystalline formations and finally to the proposed multi-zone massive injection (with the objective of generating multiple wing cracks) in naturally fractured crystalline formations. As this review does not aim at a project-by-project evaluation of the geomechanics that occur during EGS stimulation, the modified MIT definition is considered to be suitable for generating the database proposed in this study.

Geopressured and magma systems were left out from this review because they typically have been excluded from past EGS cataloguing attempts, such as those proposed by European Geothermal Energy Council (EGEC) (2012) and GtV (2013).

\section{EGS milestones}

During the last four decades, there have been some key milestones towards the development of EGS for heat production and electricity generation. The information that follows is based on the report by Tenzer (2001), supplemented by additional information:

- 1970: Proposals for the first EGS worldwide in Fenton Hill, Los Alamos, USA.

- 1973: First EGS experiments in Fenton Hill.

- 1974 to 1977: Feasibility studies for EGS projects in Japan.

- 1975: Start of preparations for the first scientific EGS pilot plant in Bad Urach, Germany.

- Since 1977: EGS feasibility studies for shallow depths at Falkenberg, Germany, Camborne School of Mines, Cornwall in the UK and Le Mayet, France.

- 1977: EGS Bad Urach - drilling starts.

- 1980 to 1986: EGS Bad Urach - deepening of the borehole to $3,488 \mathrm{~m}$ at $147^{\circ} \mathrm{C}$ and hydraulic tests for single borehole system.

- 1984 to 1985: Start of EGS; Neustadt-Glewe, as a pilot project for low enthalpy energy; to date, this is the warmest accessed hot water reservoir in Northern Germany.

- 1986: Start of the German-French EGS project at Soultz-sous-Forêts, France, as a joint European research EGS pilot plant.

- 1986 to 1991: First EGS experiments in Hijori and other locations in Japan.

- 1987: EGS Soultz - began drilling the first borehole to $2,000 \mathrm{~m}$ at $140^{\circ} \mathrm{C}$ and started the investigation of the crystalline basement in the Rhine-Graben.

- 1989: EGS Soultz - UK joins the project; formation of an industrial consortium for organized planning and operation of an EGS project in Europe.

- 1990: EGS Soultz - drilling of a second 2,000-m deep borehole and deepening of the first borehole to $3,500 \mathrm{~m}$ depth (at $160^{\circ} \mathrm{C}$ ); geothermal reservoir identification; the second borehole was used as seismic observation borehole.

- 1991 to 1996: EGS Bad Urach - deepening of the borehole to a depth of 4,445 m at a temperature of $172^{\circ} \mathrm{C}$; also performed intense borehole measurement programme.

- 1994-1995: EGS Soultz - deepening of the second borehole to a depth of 3,876 m, followed by a production test which saw the first steam production in Middle Europe from crystalline rocks; using massive stimulation and circulation tests 
with seismic monitoring and development of the downhole heat exchanger, a thermal power of $8 \mathrm{MW}$ was achieved.

- 1996: Start of deep heat mining project in Basel, Switzerland - a pilot project for EGS in a modern urban environment.

- 1996 to 1997: EGS Bad Urach - development of a downhole heat exchanger by massive hydraulic fracturing; the largest EGS created worldwide; long-term (4 months) hydraulic circulation test; a thermal power of $11 \mathrm{MW}$ was achieved.

- 1998 to 2000: EGS Soultz - deepening of the second borehole to 5,060 m at $201^{\circ} \mathrm{C}$; hydraulic stimulation and seismic monitoring.

- 2001: Start of EGS Groß-Schönebeck, Germany, which was the first in situ geothermal laboratory for developing techniques for the exploration and usage of geothermal energy.

- 2003: Start of EGS Cooper Basin, Australia - the largest demonstration EGS project in the world.

- 2003: Test of new single well concept in Genesys Horstberg, Germany.

- 2003: Start of EGS Landau - the first geothermal combined heat power plant to be connected to the grid; the one and only EGS project in a German town.

- 2004: Start of Unterhaching, Germany, the first geothermal project in the Bavarian Molasse Basin where, in addition to heat supply, electricity generation was also achieved; first Kalina power plant in Germany; first project worldwide with a private sector insurance for geological risk in deep boreholes.

- 2005: Start of EGS Paralana trying to implement an underground heat exchanger called 'heat exchanger within an insulator (HEWI)' concept (heat exchanger within the insulator) (Petratherm 2012).

- 2006/2007: Deep heat mining project in Basel stopped due to repeated severe induced seismicity events; the project was permanently abandoned in 2009.

- 2007: First binary geothermal plant in France at EGS Soultz (with ORC plant).

- 2009: New law for renewable energies in Germany - electricity generation and supply to the power net gets more financial support.

- 2009: Start of EGS GeneSys Hannover, Germany, as a single well concept.

- 2009: Start of EGS St. Gallen, Switzerland.

- 2010: Implementation of new 'side-leg' concept in the EGS project Insheim (Germany); forked injection well shall reduce induced seismicity (Insheim 2012).

- 2011: EGS GeneSys Hannover put on hold due to salt deposition in the single well.

- 2011: Guidelines for 'seismic surveillance' for Germany published by Bundesverband Geothermie.

- 2012: Switzerland decides to support deep geothermal projects.

- 2012: EGS Insheim connected to the power net.

- 2013: EGS Habanero successfully commissioned, with generation of $1 \mathrm{MW}_{\mathrm{e}}$ of power; first EGS project in Australia generating electricity.

- 2013: EGS St. Gallen drilling started.

- 2013: EGS St. Gallen put on hold due to induced seismicity events with a maximum magnitude of 3.6 on the Richter Scale; green light to proceed given by City Council 51/2 weeks later. 


\section{Systematic overview of past and present EGS projects worldwide}

The following review should not be considered exhaustive as it is based exclusively on the information available in the public domain. Yet, to the authors' knowledge, this is the first public attempt to formally collate a large database of information on EGS worldwide, from the first HDR project at Fenton Hill in 1974 to date.

The objective of this review is to present key information on past and present EGS experience worldwide, from which key lessons can be learnt for the future.

The 31 EGS projects identified during this review are classified by country, reservoir type, depth, reservoir and wellhead temperature, stimulation methods, induced seismicity and radioactivity, plant capacity, flow rate and current status.

The 31 projects are divided into four different groups:

Table 1 comprises basic information about EGS projects that are still under development. It does not include pending commercial projects that are either at the status of raising funds (e.g. Munster in Germany and Eden in the UK) or still need governmental approval.

Tables 2 and 3 present projects that are already in the power generation phase.

Table 4 gives information about experimental projects that were developed to test single phase of an EGS project rather than the whole process to generate electricity.

Table 5 presents information on projects that are aimed for electricity generation but were abandoned due to various problems. Input information was drawn from different sources available in the public domain; all of which are cited in the titles of the tables.

This grouping criteria allow the reader to have an immediate overview of past vs. current vs. future EGS activities, better appreciate the challenges faced by EGS (technical, economic and related to public acceptance), develop a feeling for the level of research and development efforts put into EGS vis-à-vis the desire to achieve worldwide commercialisation of the concept.

Note that in the tables, 'microseismic' refers to seismic activity less than 3.5 on the Richter scale and is used for those cases when no further details on recorded seismicity could be found in the literature. See the following paragraphs for more discussions on induced seismicity in EGS projects.

When 'thermal capacity' is quoted next to 'installed electrical capacity', this implies a combined heat and power project.

Under 'stimulation methods', the terms 'hydraulic fracturing', 'hydraulic', 'hydroshearing,' 'shear' and 'hydraulic stimulation' are taken directly as quoted by the cited sources. The authors of this manuscript have not performed an independent review or assessment of the specific stimulation methods implemented in or planned for each individual project, as this falls outwith the scope of this broader EGS review.

Overall, the tables above capture a detailed database of 31 EGS projects worldwide. Based on the tables, the following plots provide a way to extract trends and common characteristics of EGS.

As illustrated in Figure 1, most of the European EGS projects' reservoir/ bottomhole temperatures are lower than $165^{\circ} \mathrm{C}$, with the exception of Lardarello and Bouillante. Compared to Europe, the average EGS reservoir/bottomhole temperatures in America, Australia and Asia are higher although the well depths are comparable. Note that only 25 projects are displayed in Figure 1; the remaining 6 
Table 1 EGS projects (R\&D and commercial) still under development and not generating electricity

\begin{tabular}{|c|c|c|c|c|c|c|c|c|c|c|c|}
\hline Project & Start date & Location & $\begin{array}{l}\text { Well depth } \\
\text { (m) }\end{array}$ & $\begin{array}{l}\text { Stimulation } \\
\text { methods }\end{array}$ & Description & Operator & Current status & Rock type & $\mathrm{BHT}\left({ }^{\circ} \mathrm{C}\right)$ & $\begin{array}{l}\text { Seismic } \\
\text { event }\end{array}$ & Flow rate $(1 / s)$ \\
\hline Le Mayet $^{\mathrm{a}}$ & $\begin{array}{l}1978 \text { (Cornet } \\
\text { 2012) }\end{array}$ & $\begin{array}{l}\text { France (Cornet } \\
\text { 2012) }\end{array}$ & $\begin{array}{l}200 \text { to } 800 \\
\text { (Cornet 2012) }\end{array}$ & $\begin{array}{l}\text { Hydraulic fracturing } \\
\text { with and without } \\
\text { proppant (Cornet } \\
\text { 2012; MIT et al. } \\
\text { 2006b) }\end{array}$ & $\begin{array}{l}\text { Research } \\
\text { (Cornet 2012; } \\
\text { MIT et al. } \\
\text { 2006b) }\end{array}$ & Not known & Not known & $\begin{array}{l}\text { Granite (Cornet } \\
\text { 2012) }\end{array}$ & $\begin{array}{l}22 \text { (Wyborn } \\
\text { 2011) }\end{array}$ & $\begin{array}{l}\text { Microseismic, } \\
\text { not felt on } \\
\text { surface } \\
\text { (Cornet 2012) }\end{array}$ & $\begin{array}{l}5.2 \text { (Wyborn } \\
\text { 2011) }\end{array}$ \\
\hline $\begin{array}{l}\text { Genesys } \\
\text { Hannover }\end{array}$ & $\begin{array}{l}2009 \\
\text { (Zimmermann } \\
\text { et al. 2009) }\end{array}$ & $\begin{array}{l}\text { Germany } \\
\text { (Zimmermann } \\
\text { et al. 2009) }\end{array}$ & $\begin{array}{l}\text { 3,900 } \\
\text { (Zimmermann } \\
\text { et al. 2009) }\end{array}$ & $\begin{array}{l}\text { Hydraulic fracturing } \\
\text { (Zimmermann et al. } \\
\text { 2009) }\end{array}$ & $\begin{array}{l}\text { Demonstrate } \\
\text { single well } \\
\text { concepts } \\
\text { (Zimmermann } \\
\text { et al. 2009) }\end{array}$ & $\begin{array}{l}\text { Federal } \\
\text { Ministry of } \\
\text { Economics } \\
\text { and } \\
\text { Technology } \\
\text { (Zimmermann } \\
\text { et al. 2009) }\end{array}$ & $\begin{array}{l}\text { Salt deposition has } \\
\text { been removed (BGR } \\
\text { 2013) }\end{array}$ & $\begin{array}{l}\text { Bunter sandstone } \\
\text { (Zimmermann } \\
\text { et al. 2009) }\end{array}$ & $\begin{array}{l}160 \\
\text { (Blöscher } \\
\text { et al. 2012) }\end{array}$ & $\begin{array}{l}\text { Microseismic } \\
(1.8 \mathrm{M}) \\
(\text { Huenges } \\
2010)\end{array}$ & $\begin{array}{l}7 \text { (planned) } \\
\text { (Zimmermann } \\
\text { et al. 2009) }\end{array}$ \\
\hline $\begin{array}{l}\text { Groß } \\
\text { Schönebeck }\end{array}$ & $\begin{array}{l}2000 \\
\text { (Zimmermann } \\
\text { et al. 2009) }\end{array}$ & $\begin{array}{l}\text { Germany } \\
\text { (Zimmermann } \\
\text { et al. 2009) }\end{array}$ & $\begin{array}{l}\text { 4,309 } \\
\text { (Zimmermann } \\
\text { et al. 2009; } \\
\text { BINE 2012a) to } \\
\text { 4,400 (BINE } \\
\text { 2012a) }\end{array}$ & $\begin{array}{l}\text { Hydraulic gel } \\
\text { proppant and } \\
\text { fracturing } \\
\text { (Zimmermann et al. } \\
\text { 2009; Blöscher et al. } \\
\text { 2012; Huenges 2010) } \\
\text { thermal (ENGINE } \\
\text { 2008b), chemical } \\
\text { (Henninges et al. } \\
\text { 2012) }\end{array}$ & $\begin{array}{l}\text { 1st in situ } \\
\text { geothermal } \\
\text { laboratory, EGS } \\
\text { research } \\
\text { (Zimmermann } \\
\text { et al. 2009) }\end{array}$ & $\begin{array}{l}\text { GFZ, Schmidt } \\
+ \text { Clemens } \\
\text { GmbH + Co. } \\
\text { KG (BINE } \\
\text { 2012a) }\end{array}$ & $\begin{array}{l}\text { Production-injection } \\
\text { experiment and data } \\
\text { interpretation and } \\
\text { modelling finished } \\
\text { (Feldbusch et al. } \\
\text { 2013) }\end{array}$ & $\begin{array}{l}\text { Sandstone and } \\
\text { andesitic volcanic } \\
\text { rocks } \\
\text { (Zimmermann } \\
\text { et al. 2009; } \\
\text { Blöscher et al. } \\
\text { 2012) }\end{array}$ & $\begin{array}{l}145 \\
\text { (Blöscher } \\
\text { et al. 2012) }\end{array}$ & $\begin{array}{l}\text { Negligible } \\
\text { (max, }-1.8 \text { to } \\
-1.0 \mathrm{M}) \\
\text { (Blöscher } \\
\text { et al. 2012) }\end{array}$ & $\begin{array}{l}20 \text { (Blöscher } \\
\text { et al. 2012) }\end{array}$ \\
\hline Mauerstetten & $\begin{array}{l}2011 \text { (Schrage } \\
\text { et al. 2012a) }\end{array}$ & $\begin{array}{l}\text { Germany } \\
\text { (Schrage et al. } \\
\text { 2012a) }\end{array}$ & $\begin{array}{l}\text { 4,545 (Exorka } \\
2013 \text { ) }\end{array}$ & $\begin{array}{l}\text { Chemical (Schrage } \\
\text { et al. 2012b); } \\
\text { hydraulic } \\
\text { (Informationsportal } \\
\text { Tiefe Geothermie } \\
\text { 2013a) }\end{array}$ & $\begin{array}{l}\text { Research } \\
\text { (Schrage et al. } \\
\text { 2012b) }\end{array}$ & $\begin{array}{l}\text { Exorka GmbH, } \\
\text { GFZ, TUBAF } \\
\text { (Schrage et al. } \\
\text { 2012a) }\end{array}$ & $\begin{array}{l}\text { Seismic monitoring } \\
\text { system installed } \\
\text { (Informationsportal } \\
\text { Tiefe Geothermie } \\
\text { 2013a); next step, } \\
\text { hydraulic stimulation } \\
\text { (Informationsportal } \\
\text { Tiefe Geothermie } \\
\text { 2013a) }\end{array}$ & $\begin{array}{l}\text { Limestone } \\
\text { (Schrage et al. } \\
\text { 2012a) }\end{array}$ & $\begin{array}{l}130 \text { (Schrage } \\
\text { et al. 2012a) }\end{array}$ & Unknown & Unknown \\
\hline St. Gallen & $\begin{array}{l}2009 \\
\text { (Geothermie } \\
\text { Stadt St. } \\
\text { Gallen 2013a) }\end{array}$ & $\begin{array}{l}\text { Switzerland } \\
\text { (Geothermie } \\
\text { Stadt St. } \\
\text { Gallen 2013a) }\end{array}$ & $\begin{array}{l}\text { 4,450 } \\
\text { (Geothermie } \\
\text { Stadt St. } \\
\text { Gallen 2013a) }\end{array}$ & $\begin{array}{l}\text { Chemical and } \\
\text { hydraulic } \\
\text { (Geothermie Stadt St. } \\
\text { Gallen 2013a) }\end{array}$ & $\begin{array}{l}\text { Commercial: } \\
\text { heat and } \\
\text { power } \\
\text { (Geothermie } \\
\text { Stadt St. Gallen } \\
\text { 2013a) }\end{array}$ & $\begin{array}{l}\text { ITAG Tiefbohr } \\
\text { GmbH } \\
\text { (Geothermie } \\
\text { Stadt St. } \\
\text { Gallen 2013a) }\end{array}$ & $\begin{array}{l}\text { Production test } \\
\text { interrupted due to } \\
\text { pump failure and } \\
\text { resulting seismic } \\
\text { event (Geothermie } \\
\text { Stadt St. Gallen } \\
\text { 2013a) }\end{array}$ & $\begin{array}{l}\text { Malm, shell } \\
\text { limestone } \\
\text { (Geothermie } \\
\text { Stadt St. Gallen } \\
\text { 2013a) }\end{array}$ & $\begin{array}{l}130 \text { to } 150 \\
\text { (estimated) } \\
\text { (Geothermie } \\
\text { Stadt St. } \\
\text { Gallen } \\
\text { 2013a) }\end{array}$ & $\begin{array}{l}3.5 \mathrm{M} \\
\text { (Geothermie } \\
\text { Stadt St. } \\
\text { Gallen 2013a) }\end{array}$ & $\begin{array}{l}\text { (Geothermie } \\
\text { Stadt St. } \\
\text { Gallen 2013a) }\end{array}$ \\
\hline
\end{tabular}


Table 1 EGS projects (R\&D and commercial) still under development and not generating electricity (Continued)

\begin{tabular}{|c|c|c|c|c|c|c|c|c|c|c|c|}
\hline Newberry & $\begin{array}{l}2010 \\
\text { (Cladouhos } \\
\text { et al. 2012) }\end{array}$ & $\begin{array}{l}\text { USA } \\
\text { (Cladouhos } \\
\text { et al. 2012) }\end{array}$ & $\begin{array}{l}3,066 \text { (BLM } \\
2012)\end{array}$ & $\begin{array}{l}\text { Hydroshearing, } \\
\text { multi-zone isolation } \\
\text { techniques } \\
\text { (Cladouhos et al. } \\
\text { 2012) }\end{array}$ & $\begin{array}{l}\text { Demonstration } \\
\text { for EGS } \\
\text { stimulation/ } \\
\text { research } \\
\text { (Cladouhos } \\
\text { et al. 2012) }\end{array}$ & $\begin{array}{l}\text { AltaRock } \\
\text { Energy, } \\
\text { Davenport } \\
\text { Newberry } \\
\text { (Cladouhos } \\
\text { et al. 2012) }\end{array}$ & $\begin{array}{l}\text { Stimulation started } \\
\text { successfully } \\
\text { (Informationsportal } \\
\text { Tiefe Geothermie } \\
\text { 2012) }\end{array}$ & $\begin{array}{l}\text { Volcanic rocks } \\
\text { (Fittermann 1988) }\end{array}$ & $\begin{array}{l}315 \\
\text { (Cladouhos } \\
\text { et al. 2012) }\end{array}$ & $\begin{array}{l}\text { Microseismic } \\
\text { (Cladouhos } \\
\text { et al. 2012) }\end{array}$ & Unknown \\
\hline $\begin{array}{l}\text { Northwest } \\
\text { Geysers }\end{array}$ & $\begin{array}{l}\text { In 1980s } \\
\text { (Garcia et al. } \\
\text { 2012) }\end{array}$ & $\begin{array}{l}\text { USA (Romero } \\
\text { et al. 1995) }\end{array}$ & $\begin{array}{l}\text { 3,396 (Garcia } \\
\text { et al. 2012) }\end{array}$ & $\begin{array}{l}\text { Thermal fracturing } \\
\text { (Walters 2013) }\end{array}$ & $\begin{array}{l}\text { Demonstration/ } \\
\text { research } \\
\text { (Garcia et al. } \\
\text { 2012) }\end{array}$ & $\begin{array}{l}\text { Calpine } \\
\text { Corporation } \\
\text { (Garcia et al. } \\
\text { 2012) }\end{array}$ & $\begin{array}{l}\text { Stimulation stage ( } 5 \\
\text { MW of potential } \\
\text { production) (Walters } \\
\text { 2013) }\end{array}$ & $\begin{array}{l}\text { Metasedimentary } \\
\text { rocks (greywacke) } \\
\text { (Romero et al. } \\
\text { 1995; Garcia et al. } \\
\text { 2012) }\end{array}$ & $\begin{array}{l}\text { About } 400 \\
\text { (Garcia et al. } \\
\text { 2012) }\end{array}$ & $\begin{array}{l}\text { Microseismic } \\
\text { (0.9 to } 2.87 \\
\text { M) (Garcia } \\
\text { et al. 2012; } \\
\text { Walters 2013) }\end{array}$ & $\begin{array}{l}9.70 \text { (Garcia } \\
\text { et al. 2012) }\end{array}$ \\
\hline Paralana & $\begin{array}{l}2005 \\
\text { (Petratherm } \\
\text { 2012) }\end{array}$ & $\begin{array}{l}\text { Australia } \\
\text { (Petratherm } \\
\text { 2012) }\end{array}$ & $\begin{array}{l}4,003 \\
\text { (Petratherm } \\
\text { 2012) }\end{array}$ & $\begin{array}{l}\text { Hydraulic } \\
\text { (Petratherm 2012) }\end{array}$ & $\begin{array}{l}\text { Commercial } \\
\text { power } \\
\text { development } \\
\text { (Petratherm } \\
\text { 2012) }\end{array}$ & $\begin{array}{l}\text { Petratherm, } \\
\text { Beach Energy } \\
\text { (Petratherm } \\
\text { 2012) }\end{array}$ & $\begin{array}{l}\text { Drilling of Paralana 3, } \\
\text { submit funding } \\
\text { application } \\
\text { (Petratherm 2012) }\end{array}$ & $\begin{array}{l}\text { Metasediments, } \\
\text { granite } \\
\text { (Petratherm 2012) }\end{array}$ & $\begin{array}{l}171 \\
\text { (Petratherm } \\
\text { 2012) }\end{array}$ & $\begin{array}{l}\text { Microseismic } \\
\leq 2.6 \mathrm{M} \\
\text { (Petratherm } \\
\text { 2012) }\end{array}$ & $\begin{array}{l}\text { Up to } 6 \\
\text { (ENGINE } \\
\text { 2008b) }\end{array}$ \\
\hline
\end{tabular}

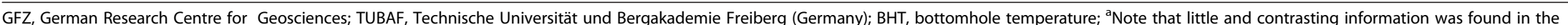
open domain concerning the project 'Le Mayet'. Some sources say that it was operational from 1984 till 1987 (Evans 2011). Others (MIT et al. 2006b) report that it was still ongoing as of 2006 and having a BHT of $33^{\circ} \mathrm{C}$ instead of the $22^{\circ} \mathrm{C}$ reported in the table. 
Table 2 Ongoing EGS projects (R\&D and commercial) generating electricity

\begin{tabular}{|c|c|c|c|c|c|c|c|c|c|}
\hline Project & Start date & Location & Well depth (m) & $\begin{array}{l}\text { Stimulation } \\
\text { methods }\end{array}$ & Description & Operator & Rock type & $\begin{array}{l}\text { Reservoir } \\
\text { temperature } \\
\left({ }^{\circ} \mathrm{C}\right)\end{array}$ & Seismic event \\
\hline \multicolumn{10}{|l|}{ A. } \\
\hline Bruchsal & $\begin{array}{l}1,983 \\
\text { (BMU2011) }\end{array}$ & $\begin{array}{l}\text { Germany } \\
\text { (BMU2011) }\end{array}$ & $\begin{array}{l}1,874 \text { to } 2,542 \\
\text { (BMU2011) }\end{array}$ & Unknown & Commercial (Enbw 2013) & EnBW, EWB (KIT 2013) & $\begin{array}{l}\text { Bunter Sandstone } \\
\text { (KIT 2013) }\end{array}$ & $\begin{array}{l}123 \\
\text { (Rettenmaier } \\
\text { 2012) }\end{array}$ & $\begin{array}{l}\text { Microseismic } \\
\text { (KIT 2013) }\end{array}$ \\
\hline Landau & $\begin{array}{l}2003(B I N E \\
2012 d)\end{array}$ & $\begin{array}{l}\text { Germany } \\
\text { (Baumgärtner } \\
\text { 2012) }\end{array}$ & $\begin{array}{l}\text { 3,170 to 3,300 } \\
\text { (Baumgärtner } \\
\text { 2012) }\end{array}$ & $\begin{array}{l}\text { No stimulation for } \\
\text { producer; hydraulic } \\
\text { for injector } \\
\text { (Baumgärtner } \\
\text { 2012) }\end{array}$ & $\begin{array}{l}\text { First implementation of EGS } \\
\text { technology in Germany (BINE } \\
2012 d) \text {; first and only EGS in } \\
\text { town in (D) (Baumgärtner 2012) }\end{array}$ & $\begin{array}{l}\text { BESTEC, Geox } \\
\text { (Baumgärtner 2012) }\end{array}$ & $\begin{array}{l}\text { Granite } \\
\text { (Lacirignola and } \\
\text { Blanc 2012) }\end{array}$ & $\begin{array}{l}159 \\
\text { (Baumgärtner } \\
\text { 2012) }\end{array}$ & $\begin{array}{l}\text { Microseismic } \\
(\leq 2.7 \mathrm{M}) \\
\text { (Baumgärtner } \\
\text { 2012), felt by } \\
\text { residents }\end{array}$ \\
\hline Insheim & $\begin{array}{l}2007 \\
\text { (Insheim } \\
\text { 2012) }\end{array}$ & $\begin{array}{l}\text { Germany } \\
\text { (Insheim } \\
\text { 2012) }\end{array}$ & $\begin{array}{l}3,600 \text { to } 3,800 \\
\text { (LGB-rlp 2012) }\end{array}$ & $\begin{array}{l}\text { Yes (Baumgärtner } \\
\text { 2012) }\end{array}$ & $\begin{array}{l}\text { New concept, side-leg injection } \\
\text { well (BINE 2012b) }\end{array}$ & $\begin{array}{l}\text { Pfalzwerke geofuture } \\
\text { GmbH (Pfalzwerke- } \\
\text { geofuture 2012; BINE } \\
\text { 2012b) }\end{array}$ & $\begin{array}{l}\text { Keuper, perm, } \\
\text { bunter } \\
\text { sandstone, } \\
\text { granite } \\
\text { (Baumgärtner } \\
\text { 2012) }\end{array}$ & $\begin{array}{l}165(\text { LGB-rlp } \\
2012)\end{array}$ & $\begin{array}{l}\text { M: } 2.0 \text { to } 2.4 \\
\text { and } \\
\text { microseismic } \\
\text { (Groos et al. } \\
\text { 2012) }\end{array}$ \\
\hline $\begin{array}{l}\text { Neustadt- } \\
\text { Glewe }\end{array}$ & $\begin{array}{l}1984(\mathrm{BMU} \\
2011)\end{array}$ & $\begin{array}{l}\text { Germany } \\
\text { (Bracke 2012) }\end{array}$ & $\begin{array}{l}\text { 2,320 (Bracke } \\
\text { 2012) }\end{array}$ & Unknown & $\begin{array}{l}\text { Commercial, pilot plant for low } \\
\text { enthalpy (BMU2011) }\end{array}$ & $\begin{array}{l}\text { WEMAG AG, Stadt } \\
\text { Neustadt-Glewe, } \\
\text { Geothermie } \\
\text { Neubrandenburg GmbH } \\
\text { (BMU2011) }\end{array}$ & $\begin{array}{l}\text { Sandstone (BMU } \\
\text { 2011) }\end{array}$ & $99($ GtV 2013) & Unknown \\
\hline Unterhaching & $\begin{array}{l}2004 \\
\text { (BMU2011) }\end{array}$ & $\begin{array}{l}\text { Germany } \\
\text { (Bracke 2012) }\end{array}$ & $\begin{array}{l}3,350 \text { to } 3,580 \\
\text { (Bracke } 2012 \text { ) }\end{array}$ & $\begin{array}{l}\text { Acidizing } \\
\text { (BMU2011) }\end{array}$ & $\begin{array}{l}\text { First Kalina power plant in } \\
\text { Germany (BINE 2012c) }\end{array}$ & $\begin{array}{l}\text { Geothermie } \\
\text { Unterhaching GmbH \& } \\
\text { Co. KG, Rödl \& Partner } \\
\text { GbR (BINE 2012c) }\end{array}$ & $\begin{array}{l}\text { Limestone } \\
\text { (Dumas 2010) }\end{array}$ & $\begin{array}{l}123(\text { Bracke } \\
2012)^{\mathrm{a}}\end{array}$ & Unknown \\
\hline Soultz & $\begin{array}{l}1987 \text { (MIT } \\
\text { et al. } \\
\text { 2006b) }\end{array}$ & $\begin{array}{l}\text { France } \\
\text { (Genter 2012) }\end{array}$ & $\begin{array}{l}5,093 \text { (MIT et al. } \\
\text { 2006d) }\end{array}$ & $\begin{array}{l}\text { Hydraulic fracturing } \\
\text { and acidizing (MIT } \\
\text { et al. 2006d) }\end{array}$ & $\begin{array}{l}\text { Research and demonstration } \\
\text { (Genter 2012) }\end{array}$ & $\begin{array}{l}\text { European cooperation } \\
\text { project (MIT et al. } \\
\text { 2006d) }\end{array}$ & $\begin{array}{l}\text { Granite (MIT et al. } \\
\text { 2006d) }\end{array}$ & $\begin{array}{l}165(B M U \\
2011)\end{array}$ & $\begin{array}{l}\text { Microseismic (M } \\
=-2 \text { to } 2.9) \\
\text { (Genter 2012) }\end{array}$ \\
\hline Bouillante & $\begin{array}{l}\text { 1963/1996 } \\
\text { (Bertini } \\
\text { et al. 2006) }\end{array}$ & $\begin{array}{l}\text { France } \\
\text { (Guadeloupe) } \\
\text { (Bertini et al. } \\
\text { 2006) }\end{array}$ & $\begin{array}{l}1,000 \text { to } 2,500 \\
\text { (Bertini et al. } \\
\text { 2006) }\end{array}$ & $\begin{array}{l}\text { Thermal cracking } \\
\text { (Bertini et al. 2006) }\end{array}$ & Commercial (Bertini et al. 2006) & $\begin{array}{l}\text { Geothermie Bouillante, } \\
\text { CFG-Services, BRGM, } \\
\text { ORKUSTOFNUN, COFOR } \\
\text { (Bertini et al. 2006) }\end{array}$ & $\begin{array}{l}\text { Volcanic lavas } \\
\text { and tuffs (Bertini } \\
\text { et al. 2006) }\end{array}$ & $\begin{array}{l}250 \text { to } 260 \\
\text { (Bertini et al. } \\
\text { 2006) }\end{array}$ & $\begin{array}{l}\text { Microseismic } \\
\text { (Sanjuan et al. } \\
\text { 2010) }\end{array}$ \\
\hline
\end{tabular}


Table 2 Ongoing EGS projects (R\&D and commercial) generating electricity (Continued)

\begin{tabular}{|c|c|c|c|c|c|c|c|c|c|}
\hline Altheim & $\begin{array}{l}1989 \\
\text { (Pernecker } \\
\text { 1999) }\end{array}$ & $\begin{array}{l}\text { Austria } \\
\text { (Bloomquist } \\
\text { 2012) }\end{array}$ & $\begin{array}{l}\text { 2,165 to 2,306 } \\
\text { (Bayerisches } \\
\text { Landesamt für } \\
\text { Wasserwirtschaft } \\
\text { 2011) }\end{array}$ & $\begin{array}{l}\text { Acidizing } \\
\text { (Pernecker 1999), } \\
\text { hydraulic } \\
\text { stimulation } \\
\text { (ENGINE 2008b) }\end{array}$ & Commercial (Pernecker 1999) & $\begin{array}{l}\text { Municipality of Altheim, } \\
\text { Terrawat (Pernecker } \\
\text { 1999) }\end{array}$ & $\begin{array}{l}\text { Limestone } \\
\text { (Bayerisches } \\
\text { Landesamt für } \\
\text { Wasserwirtschaft } \\
\text { 2011) }\end{array}$ & $\begin{array}{l}106 \\
\text { (Bloomquist } \\
\text { 2012) }\end{array}$ & Unknown \\
\hline Lardarello & $\begin{array}{l}1970 \\
\text { (Cappetti } \\
2006) \\
(1904)\end{array}$ & $\begin{array}{l}\text { Italy (ENGINE } \\
\text { 2008b) }\end{array}$ & $\begin{array}{l}2,500 \text { to } 4,000 \\
\text { (Bertini et al. } \\
\text { 2006) }\end{array}$ & $\begin{array}{l}\text { Hydraulic and } \\
\text { thermal stimulation } \\
\text { (ENGINE 2008b) }\end{array}$ & $\begin{array}{l}\text { Research and demonstration } \\
\text { (Cappetti 2006) and commercial }\end{array}$ & $\begin{array}{l}\text { ENEL Green Power } \\
\text { (Lazzarotto and Sabatelli } \\
\text { 2005) }\end{array}$ & $\begin{array}{l}\text { Metamorphic } \\
\text { rocks (ENGINE } \\
\text { 2008b) }\end{array}$ & $\begin{array}{l}300 \text { to } 350 \\
(\text { ENGINE } \\
2008 \mathrm{a})^{\mathrm{a}}\end{array}$ & $\begin{array}{l}\leq 3.0 \mathrm{M} \\
\text { (Bromley 2012) }\end{array}$ \\
\hline Coso & $\begin{array}{l}2002 \\
\text { (Häring } \\
\text { 2007) }\end{array}$ & $\begin{array}{l}\text { USA (Häring } \\
\text { 2007) }\end{array}$ & $\begin{array}{l}2,430 \text { to } 2,956 \\
\text { (Julian et al. 2009) }\end{array}$ & $\begin{array}{l}\text { Hydraulic, thermal } \\
\text { and chemical (Rose } \\
\text { et al. 2004) }\end{array}$ & $\begin{array}{l}\text { Research and development } \\
\text { (Häring 2007) }\end{array}$ & $\begin{array}{l}\text { Coso Operating } \\
\text { Company (EGS Coso } \\
\text { 2013) }\end{array}$ & $\begin{array}{l}\text { Diorite, } \\
\text { granodiorite, } \\
\text { granite (Rose } \\
\text { et al. 2004) }\end{array}$ & $\begin{array}{l}\geq 300 \text { (EGS } \\
\text { Coso 2013) }\end{array}$ & $\begin{array}{l}\leq 2.8 \text { M (Julian } \\
\text { et al. 2009) }\end{array}$ \\
\hline Desert Peak & $\begin{array}{l}2002(\mathrm{MIT} \\
2006 \mathrm{C})\end{array}$ & $\begin{array}{l}\text { USA (MIT } \\
\text { et al. 2006c) }\end{array}$ & $\begin{array}{l}\text { About 1,067 } \\
\text { (Chabora et al. } \\
\text { 2012) }\end{array}$ & $\begin{array}{l}\text { Shear, chemical, } \\
\text { hydraulic (Davatzes } \\
\text { et al. 2012) }\end{array}$ & $\begin{array}{l}\text { Research and development } \\
\text { (Davatzes et al. 2012) }\end{array}$ & $\begin{array}{l}\text { Ormat, GeothermEx (Val } \\
\text { Pierce 2011) }\end{array}$ & $\begin{array}{l}\text { Volcanic and } \\
\text { metamorphic } \\
\text { rocks (Chabora } \\
\text { et al. 2012) }\end{array}$ & $\begin{array}{l}179 \text { to } 196 \\
\text { (Chabora et al. } \\
\text { 2012) }\end{array}$ & $\begin{array}{l}\text { Microseismic: } \\
-0.03 \text { to } 1.7 \\
\text { (Chabora and } \\
\text { Zemach 2013) }\end{array}$ \\
\hline Berlín & $\begin{array}{l}2001 \\
\text { (Bommer } \\
\text { et al. 2006) }\end{array}$ & $\begin{array}{l}\text { El Salvador } \\
\text { (Rodríguez } \\
\text { 2003) }\end{array}$ & $\begin{array}{l}2,000 \text { to } 2,380 \\
\text { (Rodríguez 2008) }\end{array}$ & $\begin{array}{l}\text { Hydraulic fracturing } \\
\text { and chemical } \\
\text { (Rodríguez 2003) }\end{array}$ & $\begin{array}{l}\text { Developing EGS project in a } \\
\text { geothermal field (Rodríguez } \\
\text { 2003) }\end{array}$ & $\begin{array}{l}\text { Shell International } \\
\text { (Rodríguez 2003), LaGeo } \\
\text { (Bommer et al. 2006) }\end{array}$ & $\begin{array}{l}\text { Volcanic rocks } \\
\text { (Häring 2007) }\end{array}$ & $\begin{array}{l}183 \text { (Bommer } \\
\text { et al. 2006) }\end{array}$ & $\begin{array}{l}\leq 4.4 \mathrm{M} \\
\text { (Bommer et al. } \\
\text { 2006) }\end{array}$ \\
\hline Cooper Basin & $\begin{array}{l}2003 \\
\text { (Majer } \\
\text { et al. 2007) }\end{array}$ & $\begin{array}{l}\text { Australia } \\
\text { (Majer et al. } \\
\text { 2007) }\end{array}$ & $\begin{array}{l}4,421 \text { (Majer et al. } \\
\text { 2007) }\end{array}$ & $\begin{array}{l}\text { Hydraulic (Majer } \\
\text { et al. 2007; Holl } \\
\text { 2012) }\end{array}$ & $\begin{array}{l}\text { Largest demonstration project in } \\
\text { the world (Stephens and Jiusto } \\
\text { 2010) }\end{array}$ & $\begin{array}{l}\text { Geodynamics Ltd. } \\
\text { (Majer et al. 2007; } \\
\text { Geodynamics 2013) }\end{array}$ & $\begin{array}{l}\text { Granite (Majer } \\
\text { et al. 2007) }\end{array}$ & $\begin{array}{l}242 \text { to } 278 \\
\text { (Geodynamics } \\
\text { 2013) }\end{array}$ & $\begin{array}{l}\leq 3.7 \mathrm{M} \text { (Majer } \\
\text { et al. 2007) }\end{array}$ \\
\hline Hijiori & $\begin{array}{l}1985 \\
\text { (Sasaki } \\
1998)\end{array}$ & $\begin{array}{l}\text { Japan (Sasaki } \\
\text { 1998) }\end{array}$ & $\begin{array}{l}1,805 \text { to } 1,910 \\
\text { (Sasaki } 1998)\end{array}$ & $\begin{array}{l}\text { Hydraulic fracturing } \\
\text { (Sasaki 1998) }\end{array}$ & $\begin{array}{l}\text { Developing EGS technologies } \\
\text { (Sasaki 1998) }\end{array}$ & $\begin{array}{l}\text { Japan's new energy } \\
\text { (DiPippo 2012a), NEDO } \\
\text { (Sasaki 1998) }\end{array}$ & $\begin{array}{l}\text { Granodiorite } \\
\text { (Sasaki 1998) }\end{array}$ & $\begin{array}{l}190 \text { (DiPippo } \\
\text { 2012a) }\end{array}$ & $\begin{array}{l}\text { Microseismic } \\
\text { (Sasaki 1998) }\end{array}$ \\
\hline
\end{tabular}

${ }^{a}$ Reservoir temperature not available, BHT is used instead. 


\begin{tabular}{|c|c|c|c|c|c|c|}
\hline Project & Type of power plant & Flow rate $(I / s)$ & $\begin{array}{l}\text { Distance between } \\
\text { producer and injector } \\
(\mathrm{km})\end{array}$ & $\begin{array}{l}\text { Installed } \\
\text { electrical } \\
\text { capacity }\left(\mathrm{MW}_{\mathrm{e}}\right)\end{array}$ & $\begin{array}{l}\text { Thermal capacity } \\
\left(\mathrm{MW}_{\mathrm{th}}\right)\end{array}$ & Flow assurance problem \\
\hline \multicolumn{7}{|l|}{ B. } \\
\hline Bruchsal & Kalina cycle (BMU2011) & 28.5 (BMU2011) & 1.4 (BMU2011) & 0.55 (BMU2011) & 5.5 (GtV 2013) & $\begin{array}{l}\text { High salt contents }(100 \mathrm{~g} / \mathrm{l}) \text {; high } \mathrm{CO}_{2} \text { concentration } \\
\text { (BMU 2011) }\end{array}$ \\
\hline Landau & $\begin{array}{l}\text { Organic Rankine Cycle } \\
\text { (BINE 2012d) }\end{array}$ & $\begin{array}{l}70 \text { to } 80 \text { (Baumgärtner } \\
\text { 2012) }\end{array}$ & 1.5 (Bracke 2012) & $\begin{array}{l}\text { Up to } 3.6 \\
\text { (Baumgärtner 2012) }\end{array}$ & $\begin{array}{l}2 \text { to } 5 \text { (Baumgärtner } \\
\text { 2012) }\end{array}$ & Unknown \\
\hline Insheim & $\begin{array}{l}\text { Organic Rankine Cycle } \\
\text { (Informationsportal Tiefe } \\
\text { Geothermie 2013b) }\end{array}$ & $\begin{array}{l}65 \text { to } 85 \text { (planned) } \\
\text { (Pfalzwerke-geofuture } \\
\text { 2012) }\end{array}$ & Unknown & $\begin{array}{l}4.8 \text { (Pfalzwerke- } \\
\text { geofuture 2012) }\end{array}$ & $\begin{array}{l}6 \text { to } 10 \text { (Pfalzwerke- } \\
\text { geofuture 2012) }\end{array}$ & Unknown \\
\hline $\begin{array}{l}\text { Neustadt- } \\
\text { Glewe }\end{array}$ & $\begin{array}{l}\text { Organic Rankine Cycle } \\
\text { (Bracke 2012) }\end{array}$ & 35 (Bracke 2012) & $1.5(\mathrm{BMU} 2011)$ & 0.21 (Bracke 2012) & 4 (Bracke 2012) & $\begin{array}{l}\text { High salt content, high gas concentration } \\
\text { (Bracke 2012) }\end{array}$ \\
\hline Unterhaching & Kalina cycle (BMU 2011) & 150 (Bracke 2012) & 4.5 (Bracke 2012) & 3.36 (Bracke 2012) & 38 (Bracke 2012) & Unknown \\
\hline Soultz & $\begin{array}{l}\text { Organic Rankine Cycle } \\
\text { (BMU 2011) }\end{array}$ & 30 (BMU 2011) & 0.6 (BMU 2011) & 1.5 (BMU 2011) & $\begin{array}{l}\text { Non-scheduled (Dumas } \\
\text { 2010) }\end{array}$ & $\begin{array}{l}\text { Corrosion due to high salt contents } \\
\text { (BMU 2011) }\end{array}$ \\
\hline Bouillante & $\begin{array}{l}\text { Double and single flash } \\
\text { (Bertini et al. 2006) }\end{array}$ & 150 (Bertini et al. 2006) & 0.5 (Bertini et al. 2006) & $\begin{array}{l}15 \text { (Bertini et al. } \\
2006)\end{array}$ & Unknown & Unknown \\
\hline Altheim & $\begin{array}{l}\text { Organic Rankine cycle } \\
\text { (Bayerisches Landesamt für } \\
\text { Wasserwirtschaft 2011) }\end{array}$ & $\begin{array}{l}81.7 \text { (Bayerisches } \\
\text { Landesamt für } \\
\text { Wasserwirtschaft 2011) }\end{array}$ & $\begin{array}{l}1.7 \text { (Bayerisches } \\
\text { Landesamt für } \\
\text { Wasserwirtschaft 2011) }\end{array}$ & $\begin{array}{l}1.0 \text { (Bloomquist } \\
2012 \text { ) }\end{array}$ & $\begin{array}{l}12.4 \text { (Bayerisches } \\
\text { Landesamt für } \\
\text { Wasserwirtschaft 2011) }\end{array}$ & $\begin{array}{l}\text { Clogging by a mixture consisting of stone material } \\
\text { and bentonite (Pernecker 1999) }\end{array}$ \\
\hline Lardarello & Not known & 100 (Cappetti 2006) & $\begin{array}{l}\text { Variable: generally }>0.5 \\
\text { (ENGINE 2008a) }\end{array}$ & 700 (ENGINE 2008b) & Not known & $\begin{array}{l}\text { Highly corrosive, total loss of circulation when very } \\
\text { high permeability fracture zones are encountered } \\
\text { (ENGINE 2008b) }\end{array}$ \\
\hline Coso & Unknown & Unknown & 1.4 (Julian et al. 2009) & 240 (Karner 2005) & Unknown & Unknown \\
\hline Desert Peak & Unknown & $\begin{array}{l}100 \text { (Chabora and } \\
\text { Zemach 2013) }\end{array}$ & Unknown & $\begin{array}{l}1.7 \text { (additional) } \\
\text { (Chabora and } \\
\text { Zemach 2013) }\end{array}$ & Unknown & $\begin{array}{l}\text { Wellbore instability due to chemical stimulation } \\
\text { (Chabora et al. 2012) }\end{array}$ \\
\hline Berlín & $\begin{array}{l}\text { Binary power plant } \\
\text { (Prevost 2004) }\end{array}$ & Unknown & Unknown & $\begin{array}{l}54 \text { (Bommer } \\
\text { et al. 2006) }\end{array}$ & 56 (Rodríguez 2000) & Unknown \\
\hline Cooper Basin & Unknown & 30 (Holl 2012) & Unknown & $\begin{array}{l}1 \text { (Geodynamics } \\
\text { 2013) }\end{array}$ & Unknown & Unknown \\
\hline Hijiori & $\begin{array}{l}\text { Binary Power Plant } \\
\text { (DiPippo 2012a) }\end{array}$ & 17 (Sasaki 1998) & $\begin{array}{l}0.038 \text { to } 0.063 \\
\text { (DiPippo } 2012 \mathrm{a})\end{array}$ & 0.13 (DiPippo 2012a) & 8 (DiPippo 2012a) & $\begin{array}{l}\text { High water losses (Johansson et al. 1993), precipitation } \\
\text { of anhydrite (DiPippo 2012a) }\end{array}$ \\
\hline
\end{tabular}


projects (St. Gallen, Fjällbacka, Falkenberg, The Southeast Geysers, Basel and Bad Urach) are excluded because reservoir/bottomhole temperature data could not be found in the public domain or are only estimated in the case of St. Gallen.

The relationship shown in Figure 2 points out that most EGS activities are operated at flow rates lower than $40 \mathrm{l} / \mathrm{s}$. Note that only 20 projects are displayed in Figure 2; the remaining 11 projects (Genesys Hannover, Insheim, Mauerstetten, Newberry, Coso, Berlín, Falkenberg, The Southeast Geysers, Basel, Bad Urach and St. Gallen) are excluded because flow rate data could not be found in the public domain.

Figure 3 displays EGS projects classified on the basis of rock types. Although it appears that EGS activities can be implemented in any of the three major groups of rocks on earth, most projects are developed in igneous rocks, following the original HDR concept.

The recorded maximum magnitudes of induced seismic events associated with the development of EGS projects worldwide are shown in Figure 4. Originally, the Richter scale was developed as a mathematical device to compare local earthquake sizes. The magnitude is defined as the logarithm of the wave amplitude recorded by seismographs. At that time, the smallest measurable earthquakes were assigned with values close to zero. However, due to the higher accuracy of modern seismographs, the Richter scale now measures earthquakes having negative magnitudes. Majer et al. (2007) reported that '...To date, the maximum observed earthquakes attributed to EGS operations have been magnitude 3.0 to 3.7 and the largest geothermal injection-related event was magnitude 4.6'. Later, Majer et al. (2013) also stated that for EGS, earthquakes are typically smaller than M 3.5 (M representing the momentum magnitude in this context). According to EGEC (2013), microseismic activity is less than 3.5 on the Richter scale. Only the projects with published induced seismic magnitude are displayed in Figure 4; the remaining 16 projects (Le Mayet, Mauerstetten, Newberry, Bruchsal, Neustadt-Glewe, Unterhaching, Bouillante, Altheim, Hijiori, Genesys Horstberg, Fjällbacka, Fenton Hill, Ogachi, Bad Urach, Falkenberg and The Southeast Geysers), most of which have been reported to suffer from microseismicity, are omitted due to lack of explicit seismic data.

Stimulation methods that are applied in EGS developments are summarized in Figure 5, which reveals that hydraulic stimulation is the most commonly used method, independently of the rock type concerned. In addition, there are relatively few cases where chemical or thermal stimulation technologies are applied. This often leads to the assumption that the EGS definition only applies to hydraulically fractured systems.

The installed electrical and thermal capacity of EGS projects are summarized in Figure 6. Since EGS is still a developing concept, the database contains only 14 projects carried out with electricity generation. Note that the thermal capacities of Bouillante, Soultz, Lardarello, Desert Peak, Cooper Basin and Coso are missing as data could not be found in the public domain. The variation of production scale causes great capacity differences among the projects.

Figure 7 shows the rock type and well depth of all the studied EGS projects worldwide. 


\section{Results and discussions}

From the information provided in the tables and the plots shown earlier, it appears that EGS projects currently under development are still on the learning curve, overcoming problems, gaining experience and trying to introduce advanced technology; the projects already concluded provide relevant history and analogy for upcoming developments and the projects that have been temporarily halted or abandoned give an insight into issues that must be avoided in the future.

Below are field cases where breakthrough methodologies were first implemented to validate the EGS concept. Unplanned events and issues that needed addressing in order to ensure feasibility and commerciality of EGS are discussed, and the corresponding lessons learnt are highlighted.

- The 'Paralana' project will use a new concept called the HEWI (Petratherm 2012).

- The 'Genesys' project was the first project worldwide testing a single well concept. Technical feasibility of the concept was proved by the 'Genesys Horstberg' project. The subsequent 'Genesys Hannover' aimed to use geothermal energy to heat the building complex of the Geozentrum Hannover (Tischner et al. 2010). The project has currently solved the problem of salt deposition, which has led to a suspension of the production test (Genesys 2012). This single well concept has the advantage of lower drilling costs as only one wellbore is needed to be drilled. However, since the circulating fluid moves through fractures, it is in direct contact with the rock formation, which leads to salt deposition risk. This experience has taught the geothermal community that flow assurance needs to be addressed ahead of time to prevent issues triggered by the chemical interaction between the injected fluid and the receiving rock, which can impair the overall success of an EGS project.

- The 'Groß Schönebeck' project is an important pilot for the development of geothermal technologies in Europe as an in situ laboratory was installed in one of the boreholes. The reservoir can be investigated by logging tools during production using a special Y-tool which is attached to the production string (Henninges et al. 2012). This system allows measurements with electrical tools and fibre-optic distributed temperature sensing. However, the data transfer to surface is problematic and can only be done discontinuously (Huenges 2013). This suggests that further technology advances are needed in the area of well logging for this type of applications. Using a newly developed fluid monitoring system, fluid physicochemical properties were measured online and in situ (Feldbusch et al. 2013). Worldwide, it is the only facility for the investigation of sedimentary large-scale structures under natural conditions. A 7-day long-term production-injection experiment between both boreholes to investigate the sustainability of the reservoir was completed in April 2012 (Feldbusch et al. 2013), and results, data interpretation and modelling of the experiments have been presented by Feldbusch et al. (2013), Cherubini et al. (2013) and Noack et al. (2013). A corrosion test will permit the verification of the long-term reliability of the system's components Bine 4 (2012). A thermal fluid loop as the initial phase of fluid production was established and continuously operated for 7 days (Feldbusch et al. 2013). 
Table 4 Concluded experimental EGS projects (without power generation)

\begin{tabular}{|c|c|c|c|c|c|c|c|c|c|c|c|}
\hline Project & Description & $\begin{array}{l}\text { Start } \\
\text { date }\end{array}$ & Location & Rock type & $\begin{array}{l}\text { Reservoir } \\
\text { temperature } \\
\left({ }^{\circ} \mathrm{C}\right)\end{array}$ & $\begin{array}{l}\text { Well } \\
\text { depth } \\
\text { (m) }\end{array}$ & Stimulation methods & $\begin{array}{l}\text { Seismic } \\
\text { event }\end{array}$ & $\begin{array}{l}\text { Fluid } \\
\text { temperature } \\
\left({ }^{\circ} \mathrm{C}\right)\end{array}$ & End date & $\begin{array}{l}\text { Flow rate } \\
(\mathrm{I} / \mathrm{s})\end{array}$ \\
\hline Falkenberg & $\begin{array}{l}\text { Investigation of } \\
\text { hydraulic fracturing at } \\
\text { shallow depth } \\
\text { (Tenzer 2001) }\end{array}$ & $\begin{array}{l}1977 \\
\text { (Tenzer } \\
\text { 2001) }\end{array}$ & $\begin{array}{l}\text { Germany } \\
\text { (Tenzer } \\
\text { 2001) }\end{array}$ & $\begin{array}{l}\text { Granite (MIT } \\
\text { et al. 2006e) }\end{array}$ & $\begin{array}{l}13.5 \\
\text { (Kappelmeyer } \\
\text { and Jung } \\
\text { 1987) }\end{array}$ & $\begin{array}{l}500 \\
\text { (Tenzer } \\
\text { 2001) }\end{array}$ & Hydraulic fracturing (Tenzer 2001) & $\begin{array}{l}\text { Microseismic } \\
\text { (MIT et al. } \\
\text { 2006e) }\end{array}$ & Unknown & $\begin{array}{l}1986 \\
\text { (Tenzer } \\
\text { 2001) }\end{array}$ & $\begin{array}{l}0.2 \text { to } 7 \\
\text { (Kappelmeyer } \\
\text { and Jung } \\
\text { 1987) (test) }\end{array}$ \\
\hline $\begin{array}{l}\text { Genesys } \\
\text { Horstberg }\end{array}$ & $\begin{array}{l}\text { Testing of new single } \\
\text { well concepts at an } \\
\text { abandoned gas well } \\
\text { (BGR 2012a) }\end{array}$ & $\begin{array}{l}2003 \\
\text { (BGR } \\
2012 b)\end{array}$ & $\begin{array}{l}\text { Germany } \\
\text { (BGR } \\
\text { 2012a) }\end{array}$ & $\begin{array}{l}\text { Sedimentary } \\
\text { (BGR 2012a) }\end{array}$ & $\begin{array}{l}150(\text { ENGINE } \\
2012)\end{array}$ & $\begin{array}{l}3,800 \\
\text { (ENGINE } \\
2012)\end{array}$ & Hydraulic fracturing (BGR 2012a) & $\begin{array}{l}\text { No measured } \\
\text { event (Kreuter } \\
\text { 2011) }\end{array}$ & $\begin{array}{l}115 \text { (Tenzer } \\
\text { 2001) }\end{array}$ & $\begin{array}{l}2007 \\
\text { (estimation) } \\
\text { (BGR 2012b) }\end{array}$ & $\begin{array}{l}10 \text { to } 20 \\
\text { (Tischner et al. } \\
\text { 2010) }\end{array}$ \\
\hline Fjällbacka & $\begin{array}{l}\text { Experimental project } \\
\text { (Portier et al. 2007) }\end{array}$ & $\begin{array}{l}1984 \\
\text { (Jupe } \\
\text { et al. } \\
\text { 1992) }\end{array}$ & $\begin{array}{l}\text { Sweden } \\
\text { (Portier } \\
\text { et al. } \\
\text { 2007) }\end{array}$ & $\begin{array}{l}\text { Granite } \\
\text { (Portier et al. } \\
\text { 2007) }\end{array}$ & $\begin{array}{l}16 \text { (Wallroth } \\
\text { et al. 1999) }\end{array}$ & $\begin{array}{l}70 \text { to } \\
500 \\
\text { (Jupe } \\
\text { et al. } \\
1992 \text { ) }\end{array}$ & $\begin{array}{l}\text { Hydraulic fracturing and acidizing } \\
\text { (Portier et al. 2007) }\end{array}$ & $\begin{array}{l}\text { Microseismic } \\
\text { (Wallroth et al. } \\
\text { 1999) }\end{array}$ & Unknown & $\begin{array}{l}1995 \\
\text { (Wallroth } \\
\text { et al. 1999) }\end{array}$ & $\begin{array}{l}0.9 \text { to } 1.8 \\
\text { (Wallroth } \\
\text { et al. 1999) }\end{array}$ \\
\hline Rosemanowes & $\begin{array}{l}\text { Experimental project } \\
\text { (MIT 2006f) }\end{array}$ & $\begin{array}{l}1977 \\
\text { (MIT } \\
2006 f)\end{array}$ & $\begin{array}{l}\text { UK (MIT } \\
\text { 2006f) }\end{array}$ & $\begin{array}{l}\text { Granite (MIT } \\
\text { 2006f) }\end{array}$ & $\begin{array}{l}79 \text { to } 100 \\
\text { (MIT 2006f) }\end{array}$ & $\begin{array}{l}2,000 \text { to } \\
2,600 \\
\text { (MIT } \\
2006 \mathrm{f})\end{array}$ & $\begin{array}{l}\text { Hydraulic fracturing (MIT 2006f), } \\
\text { viscous gel stimulation (Parker } \\
\text { 1999), placement of proppants in } \\
\text { joints (Parker 1999) }\end{array}$ & $\begin{array}{l}\text { Max. } \\
\text { magnitude, } \\
3.1 \text { (Bromley } \\
\text { and Mongillo } \\
\text { 2008) }\end{array}$ & $\begin{array}{l}54.2 \text { to } 80 \\
\text { (Richards } \\
\text { et al. 1992) }\end{array}$ & $\begin{array}{l}1992 \text { (MIT } \\
\text { 2006f) }\end{array}$ & $\begin{array}{l}4 \text { to } 25 \text { (MIT } \\
2006 f)\end{array}$ \\
\hline Fenton Hill & $\begin{array}{l}\text { First EGS in the world } \\
\text { (MIT 2006g) }\end{array}$ & $\begin{array}{l}1974 \\
(\mathrm{MIT} \\
2006 \mathrm{~g})\end{array}$ & $\begin{array}{l}\text { USA (MIT } \\
\text { 2006g) }\end{array}$ & $\begin{array}{l}\text { Crystalline } \\
\text { rock (Brown } \\
\text { 2009) }\end{array}$ & $\begin{array}{l}200 \text { to } 327 \\
\text { (MIT 2006g) }\end{array}$ & $\begin{array}{l}2,932 \text { to } \\
4,390 \\
\text { (MIT } \\
2006 \mathrm{~g} \text { ) }\end{array}$ & Hydraulic fracturing (MIT 2006g) & $\begin{array}{l}\text { Microseismic } \\
\text { (Brown 1995) }\end{array}$ & $\begin{array}{l}180 \text { to } 192 \\
\text { (MIT 2006g) }\end{array}$ & $\begin{array}{l}1993 \text { (MIT } \\
\text { 2006g) }\end{array}$ & $\begin{array}{l}10.6 \text { to } 18.5 \\
\text { (MIT 2006g) }\end{array}$ \\
\hline Ogachi & $\begin{array}{l}\text { Test run EGS project } \\
\text { in shallow depth } \\
\text { (Kaieda et al. 2005) }\end{array}$ & $\begin{array}{l}1989 \\
\text { (Kaieda } \\
\text { et al. } \\
\text { 2005) }\end{array}$ & $\begin{array}{l}\text { Japan } \\
\text { (Kaieda } \\
\text { et al. } \\
\text { 2005) }\end{array}$ & $\begin{array}{l}\text { Granodiorite } \\
\text { (Kaieda et al. } \\
\text { 2010) }\end{array}$ & $\begin{array}{l}60 \text { to } 228 \\
\text { (Kaieda et al. } \\
\text { 2005) }\end{array}$ & $\begin{array}{l}400 \text { to } \\
1100 \\
\text { (Kaieda } \\
\text { et al. } \\
\text { 2005) }\end{array}$ & $\begin{array}{l}\text { Multiple wells with multiple } \\
\text { fracture zones (Kaieda et al. } \\
\text { 2005); hydraulic (Kaieda et al. } \\
\text { 2005) }\end{array}$ & $\begin{array}{l}\text { Few } \\
\text { microseismic } \\
\text { (Kaieda et al. } \\
\text { 2010) }\end{array}$ & $\begin{array}{l}160 \text { (test } \\
\text { result) (Kaieda } \\
\text { et al. 2005) }\end{array}$ & $\begin{array}{l}2002 \\
\text { (Kaieda } \\
\text { et al. 2005) }\end{array}$ & $\begin{array}{l}6.7 \text { to } 20 \\
\text { (Kaieda et al. } \\
\text { 2005) (test) }\end{array}$ \\
\hline
\end{tabular}


- The Altheim project in Austria uses a special working fluid, which was never used before - a non-flammable, non-corrosive fluid with no ozone depletion activity (Bloomquist 2012).

- The 'Fenton Hill' project was the first attempt to extract geothermal energy form hot dry rocks with low permeability in the history of EGS (MIT et al. 2006g). One of the main lessons learnt from the Fenton Hill project is that an engineered hot reservoir should first be created from the preliminary borehole and then by connecting the enhanced reservoir and the injection borehole with the production boreholes (Brown 2009).

- The 'Rosemanowes Quarry' project in the UK stemmed directly from the positive results from Fenton Hill. One of the most significant lessons learnt from this project is that natural fractures and engineered fractures are almost unrelated. The natural fracture network plays a more important role compared with hydraulically enhanced fractures (MIT et al. 2006f). Also, as reported by Jung (2013), until then, the basement had been regarded as a competent rock mass, realizing that in reality, the basement contains open natural fractures even at great depth led to the abandonment of the HDR multi-fracture concept and the adoption of the hydraulic stimulation EGS concept.

- The 'Fjällbacka' project in Sweden gives similar conclusions to the Rosemanowes project, i.e. that naturally fractured systems dictate the results of reservoir stimulation (Wallroth et al. 1999).

- The 'Falkenberg' project began in 1976 and was planned as a test site for HDR at shallow depths to better understand the mechanical and hydraulic properties of fractures (Kappelmeyer and Jung 1987). A power generation phase was never intended.

- The 'Ogachi' project in Japan, a five-spot well pattern (four producers, one injector), was planned to be used for geothermal energy extraction from a shallow depth reservoir, but due to financial problems, the multiple production well system was not tried out. However, several basic technologies were successfully developed for general EGS activities through the project, which were later applied in another EGS programme in the Cooper Basin, South Australia in 2002 (Kaieda et al. 2005).

- The 'Basel' project in Switzerland saw induced seismic events - some exceeding 3.0 in magnitude - which led to its suspension (Ladner and Häring 2009). The Basel area has a history of natural seismic activity; the city was severely damaged by a 6.7 magnitude earthquake in 1356, the largest seismic event ever recorded in Central Europe (Giardini 2009). However, following a 3-year study after the seismic events recorded in connection with the geothermal project activities, the Basel project was cancelled. Induced seismicity associated with water injection and particularly hydraulic fracturing activities (due to changing stress patterns in reservoir rocks) has caused wide concern among the public (Majer et al. 2011).

- The 'Insheim' project has also had issues of induced seismicity. A so-called side-leg concept for the injection well was implemented to solve the problem (BINE 2012b). This concept enables pressure distribution during fluid injection over two separated ends of the injection well, thus minimizing the risk of induced seismicity. However, in 2013, another induced seismic event with a magnitude of 2.0 on the Richter scale 
Table 5 Abandoned or on hold EGS projects

\begin{tabular}{|c|c|c|c|c|c|c|c|c|c|c|}
\hline Project & Operator & Description & Start date & Location & Rock type & Stimulation methods & Seismic event & $\begin{array}{l}\text { Well } \\
\text { depth (m) }\end{array}$ & End date & $\begin{array}{l}\text { Reasons of } \\
\text { abandonment }\end{array}$ \\
\hline Bad Urach & $\begin{array}{l}\text { Forschungs- } \\
\text { Kollegium Physik } \\
\text { des Erdkörpers } \\
\text { (MIT 2006h) }\end{array}$ & $\begin{array}{l}\text { EGS pilot by one } \\
\text { borehole only (Tenzer } \\
\text { 2001) }\end{array}$ & $\begin{array}{l}1977 \text { (Tenzer } \\
\text { 2001), } 2006 \\
\text { (Wyborn } \\
\text { 2011) }\end{array}$ & $\begin{array}{l}\text { Germany } \\
\text { (Tenzer } \\
\text { 2001) }\end{array}$ & $\begin{array}{l}\text { Gneiss } \\
\text { (Tenzer et al. } \\
\text { 2000) }\end{array}$ & $\begin{array}{l}\text { Hydraulic fracturing } \\
\text { (Schanz et al. 2003) }\end{array}$ & $\begin{array}{l}\text { Microseismicity } \\
\text { (Schanz et al. } \\
\text { 2003) }\end{array}$ & $\begin{array}{l}\text { 3,334 to } \\
4,445 \\
\text { (Schanz } \\
\text { et al. 2003) }\end{array}$ & $\begin{array}{l}1981 \text { (MIT } \\
\text { 2006i), } 2008 \\
\text { (Wyborn } \\
\text { 2011) }\end{array}$ & $\begin{array}{l}\text { Torn off bore rods in } \\
\text { borehole (Wyborn } \\
\text { 2011) }\end{array}$ \\
\hline Basel & $\begin{array}{l}\text { Geopower Basel } \\
\text { (Romano 2009) }\end{array}$ & $\begin{array}{l}\text { Planning to develop } \\
\text { EGS project (Ladner and } \\
\text { Häring 2009) }\end{array}$ & $\begin{array}{l}1996 \\
\text { (Giardini } \\
\text { 2009) }\end{array}$ & $\begin{array}{l}\text { Switzerland } \\
\text { (Romano } \\
\text { 2009) }\end{array}$ & $\begin{array}{l}\text { Granite } \\
\text { (Ladner and } \\
\text { Häring 2009) }\end{array}$ & $\begin{array}{l}\text { Hydraulic fracturing } \\
\text { (Ladner and Häring } \\
\text { 2009) }\end{array}$ & $\begin{array}{l}\text { Frequent } \\
\text { earthquakes } \\
\text { (including } 3.4 \mathrm{M}) \\
\text { (Romano 2009) }\end{array}$ & $\begin{array}{l}5,000 \\
\text { (Romano } \\
\text { 2009) }\end{array}$ & $\begin{array}{l}2009 \\
\text { (Giardini } \\
\text { 2009) }\end{array}$ & $\begin{array}{l}\text { Induced seismicity } \\
\text { exceeding } \\
\text { acceptable levels } \\
\text { (Giardini 2009) }\end{array}$ \\
\hline $\begin{array}{l}\text { The } \\
\text { Southeast } \\
\text { Geysers }\end{array}$ & $\begin{array}{l}\text { AltaRock Energy } \\
\text { (Romano 2009) }\end{array}$ & $\begin{array}{l}\text { Redrill a well for EGS } \\
\text { demonstration project } \\
\text { (AltaRock Energy Inc. } \\
\text { 2012) }\end{array}$ & $\begin{array}{l}2008 \text { (Cotler } \\
\text { 2009) }\end{array}$ & $\begin{array}{l}\text { USA } \\
\text { (Romano } \\
\text { 2009) }\end{array}$ & $\begin{array}{l}\text { Greywacke } \\
\text { (AltaRock } \\
\text { Energy Inc. } \\
\text { 2012) }\end{array}$ & $\begin{array}{l}\text { Multiple fractures zones } \\
\text { in wells (planned) } \\
\text { (AltaRock Energy Inc. } \\
\text { 2012) }\end{array}$ & $\begin{array}{l}\text { Induced seismicity } \\
\text { risk (Romano } \\
\text { 2009) }\end{array}$ & $\begin{array}{l}1,341 \\
\text { (AltaRock } \\
\text { Energy Inc. } \\
\text { 2012) }\end{array}$ & $\begin{array}{l}2009 \\
\text { (AltaRock } \\
\text { Energy Inc. } \\
\text { 2012) }\end{array}$ & $\begin{array}{l}\text { Wellbore collapsing } \\
\text { and induced } \\
\text { seismicity risk } \\
\text { (Romano 2009) }\end{array}$ \\
\hline
\end{tabular}




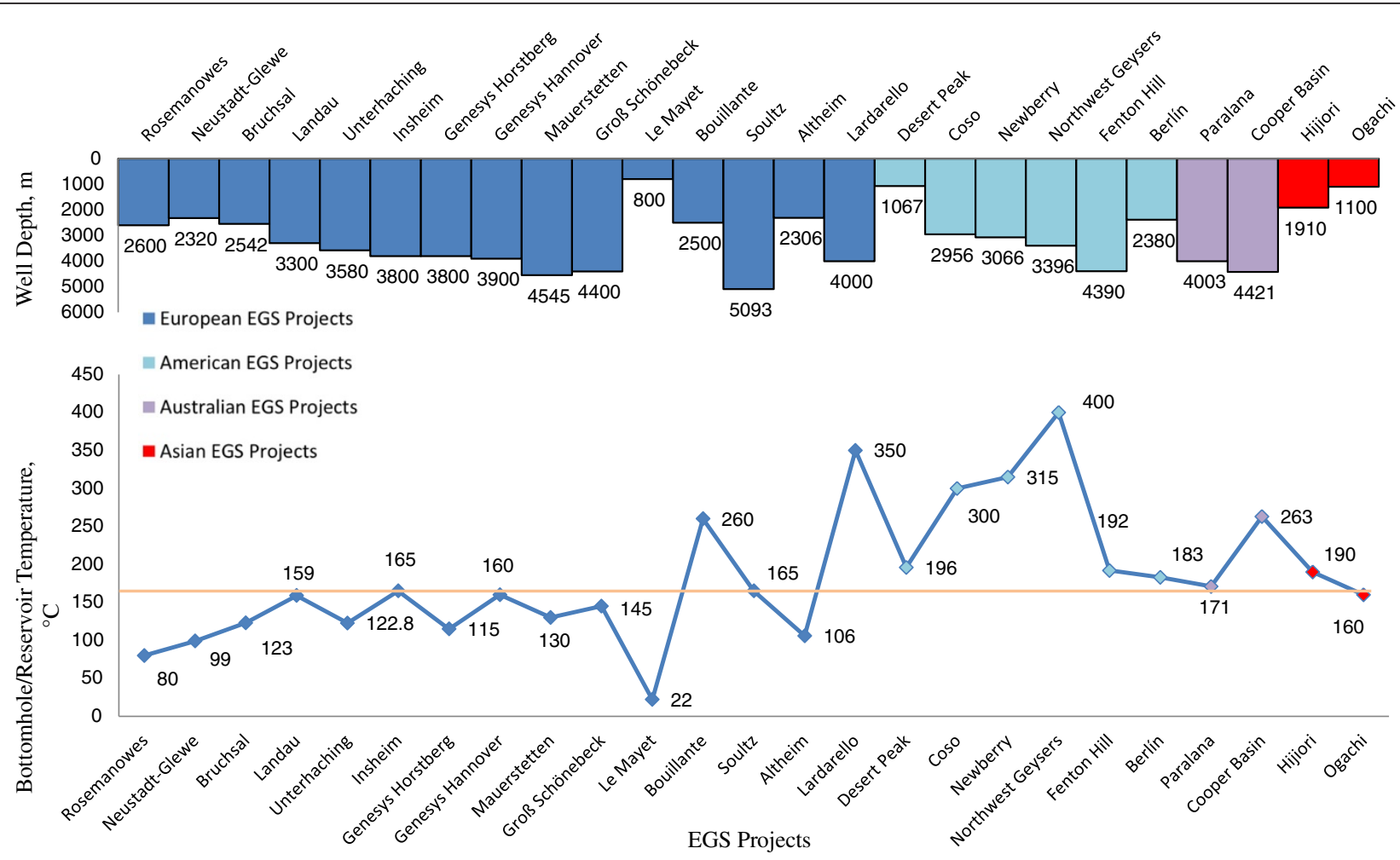

Figure 1 Worldwide EGS projects' reservoir/bottomhole temperature vs. depth. 


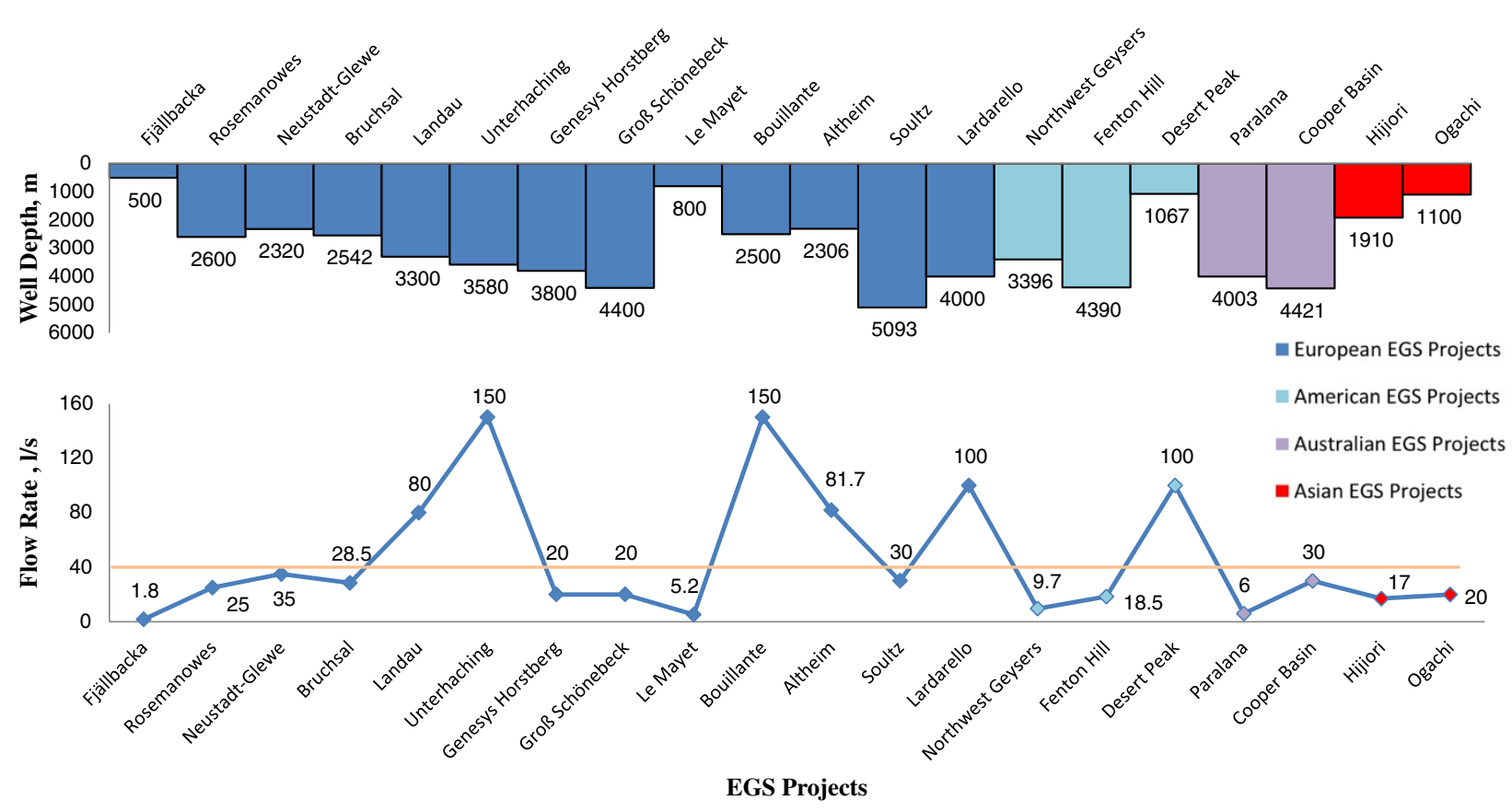

Figure 2 Worldwide EGS projects' flow rate vs. depth. 


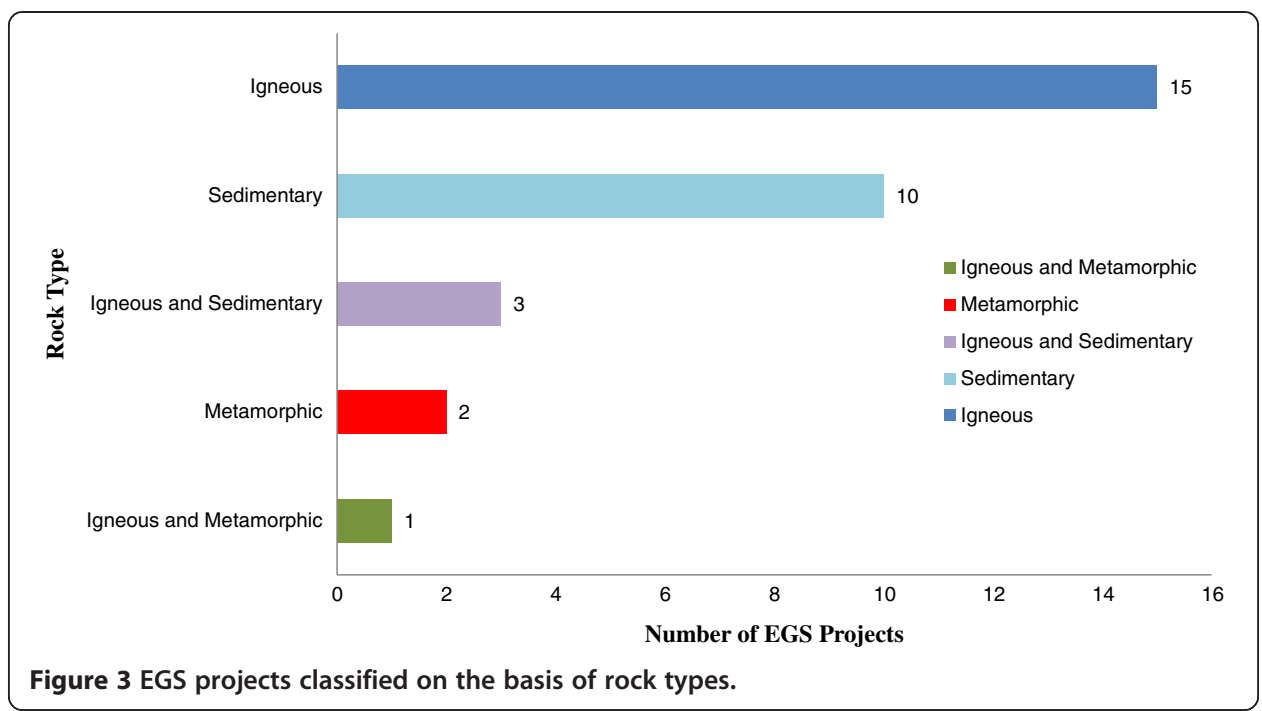

occurred due to a water circulation stop during a reparation phase of the defective production pump (Geothermie-Pfalz 2013).

- The 'Landau' project is the first EGS project in a town in Germany, which is facing similar problems to Basel. Seismic events of 2.7 in magnitude took place in 2009, which resulted to the temporary suspension of the operations. The project was restarted after purchasing $€ 50$ million of annual liability insurance to cover potential seismic damages (DiPippo 2012b). As a consequence of these events, water has to be reinjected at a reduced pressure to avoid induced seismicity, resulting in reduced power generation. The problem is planned to be tackled by implementing in 2013 the same side-leg concept that was used in Insheim (BINE 2012b).

- The 'Soultz-sous-Fôrets' project in France has allowed significant experience to be gained by several countries who participated in this joint project. Many

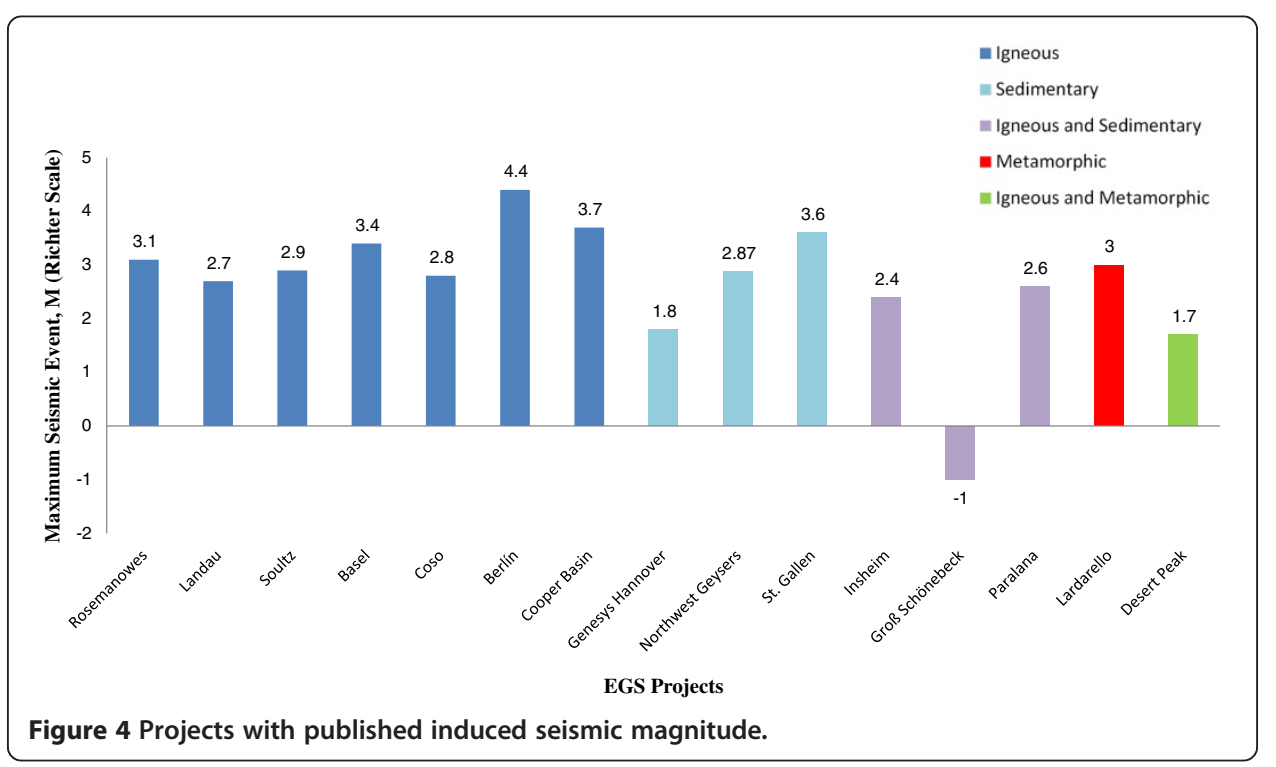


experiments were conducted during the first 21 years of the project's life before the power plant was built. Different stimulation techniques, such as hydraulic fracturing with and without proppants and chemical stimulation were applied. Chemical stimulation has resulted in less seismic activity than other methods. Change of hydraulic parameters due to fracturing has resulted in an instantaneous variation of seismic activity. Seismic events with magnitudes greater than 2 have occurred during the shut-in phase. Although minor damages were caused by this EGS project, it did generate concern among the local population.

Microseismic monitoring has become an indispensable technology for the acceptance of EGS developments as it is the case for other applications of hydraulic fracturing and high-pressure water circulation (e.g. the exploitation of unconventional oil and gas resources). The experience gained from preliminary projects has led to a common view that induced seismicity associated with EGS activities can halt further development of this concept particularly in densely populated areas. More recently, though, despite the 3.6 magnitude seismicity induced by well control operations during drilling in St. Gallen, the city council decided to continue with the project and complete the first drilling phase (Geothermie Stadt St. Gallen 2013b).

- The 'Bad Urach' project suspended operations because of financing problems arising from a 'difficult geologic situation' at the well site, which indicated that this project would be unprofitable (DiPippo 2012b).

- The 'Geysers' project was abandoned due to drilling difficulties and the risk of increasing seismic activity (AltaRock Energy Inc. 2012).

It is worth mentioning here that according to Gebo NDS (2012b), drilling expenditure is the highest component in the development costs of an EGS project and can vary from $42 \%$ to $90 \%$ of the overall capital costs.

As mentioned earlier, hydraulic stimulation is the most commonly used technique for improving the permeability of a geothermal reservoir. Some of the world's EGS projects

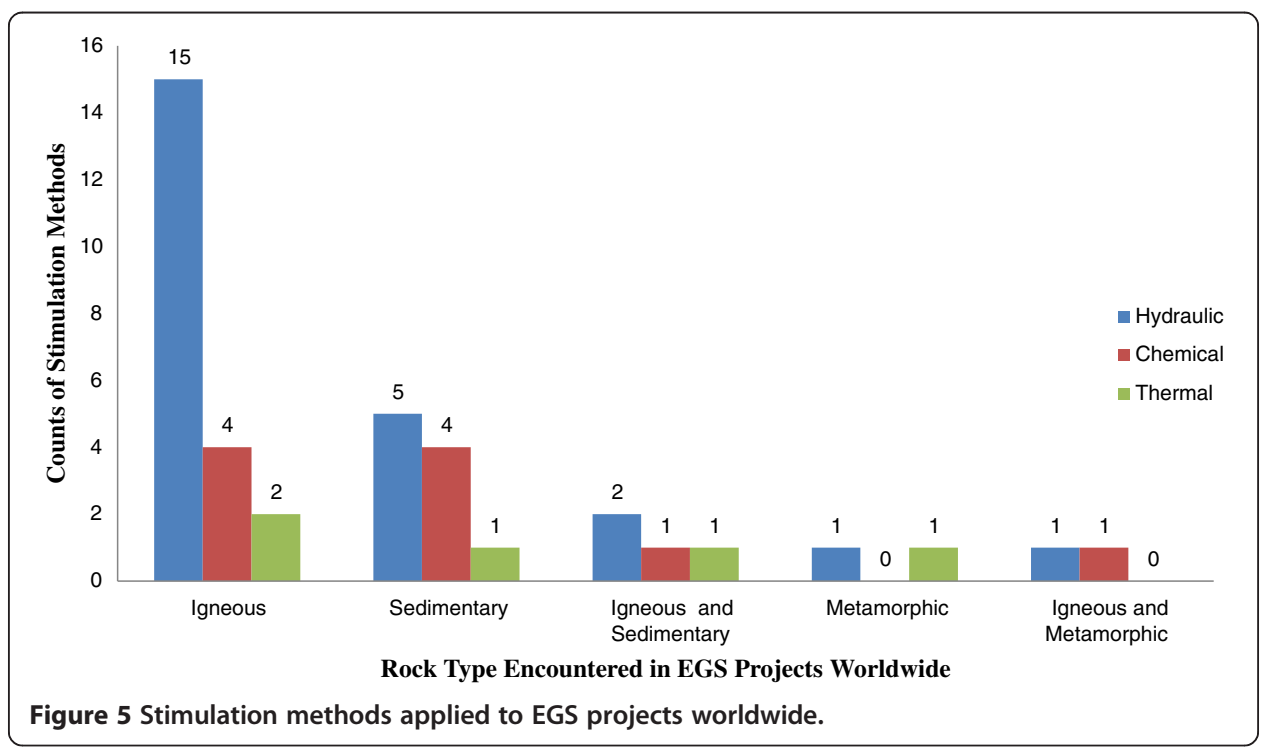




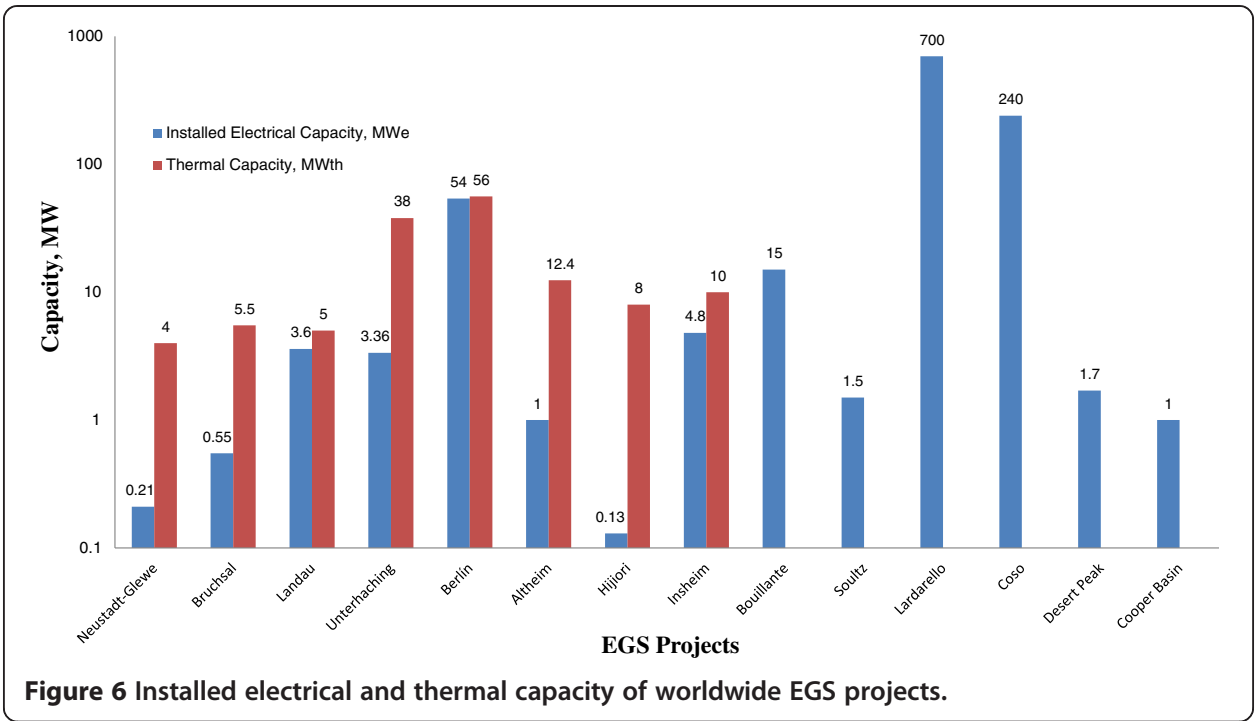

can extract geothermal energy from naturally fractured reservoirs, such as Northwest Geysers, Landau, Insheim, Urach, Bruchsal, Soultz-sous-Fôrets, Fjällbacka, Hijiori, Rosemanowes, Falkenberg and Newberry. The pre-existing naturally fractured networks can be stimulated by low pressure that is just above the critical pressure of shear failure (hydraulic shearing). However, the process of hydraulic fracturing, which uses injected water at high pressure to crack the rocks, is also frequently used especially in granite. Compared with hydraulic fracturing with high injection pressure, hydraulic shearing can easily crack rocks with low pressure and keep the fractures open without requiring a propping agent. Chemical stimulation, which is most applicable in carbonate rocks or used to dissolve carbonate cement in sandstone formations, along with thermal stimulation has also proved to be effective in some cases. However, there is relatively little literature concerning the application of chemical and thermal stimulation technologies in EGS projects.

Other issues associated with EGS stimulation are related to the potentially harmful effects on the surrounding environment. There has been public concern for the

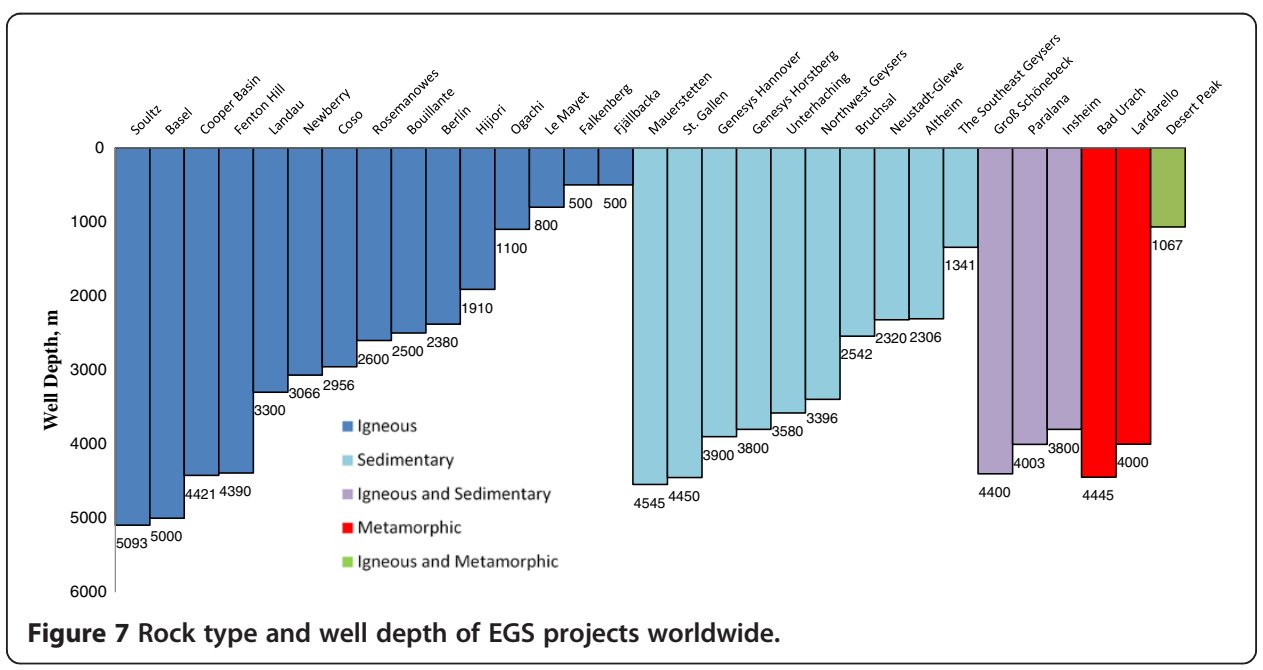


components of fracturing fluids that could represent a threat to drinking water sources. However, operators argue that EGS projects rarely require additives and chemicals (e.g. tracers, diverters, proppants) in fracturing fluids. When additives are necessary, then non-toxic chemicals are first considered. Also, deeply buried EGS reservoirs usually do not have a connection to near-surface groundwater aquifers, which would reduce the likelihood of contaminating drinking water (Regenspurg and Blöcher 2012).

Radioactivity is another problem emerging from EGS activities, which is caused by interaction between the geothermal fluid and certain formations containing radioactive elements. In general, the content of radionuclides in acidic magmatic rocks is higher compared to that in sedimentary rocks. Uranium and thorium are the most common radioactive elements found in granites. High reservoir temperature in EGS projects increases the solubility of radionuclides, which results in higher concentrations of these nuclides in the geothermal fluid. When the fluid is produced, the corresponding temperature reduction and pressure decrease in the surface facilities and causes deposition of scale, which leads to health, safety and environment problems. However, compared to other conventional energy production (e.g. oil and gas industry), the radioactivity occurring in EGS is likely to be very small (Battye and Ashman 2009). Radiation exposure of workers during the scale removal is avoided by using appropriate personal protection equipment. In general, the radiation exposure to the public is limited because long-lived natural radionuclides are not released during the operation of a geothermal power plant when the geothermal fluid is re-injected into the reservoir (Feige and Roloff 2012).

Almost all running EGS projects in the power generation phase utilize binary power plants. Binary systems use geothermal fluids with low temperature in the primary loop to vaporize working fluids with low boiling point that are used in the secondary loop to activate turbine-generator machine.

At present, two types of binary systems exist in the market: the organic Rankine cycle (ORC) which uses organic working fluids (e.g. propane or isobutane) and the Kalina Cycle which uses a mixture of two substances as the working fluid (e.g. water and ammonia). The advantage of the Kalina cycle over the ORC is that the abovementioned mixture boils at variable temperatures, which in turn creates higher efficiency at a certain inlet temperature, unlike the pure chemicals that are used in ORC (Clauser 2006). The disadvantages of the Kalina system are the challenge of fine tuning the plant operation and the tendency of the ammonia-water mixtures to prematurely condense during expansion. Hence, the majority of the EGS projects implemented so far tend to use ORC power plants.

The flow rate recovered with EGS projects is crucial in dictating the success of a project. It needs to be high to ensure the project's economic viability. Yet if the rate is too high, there may not be sufficient 'residence time' for the circulating medium in the reservoir to extract enough heat from the rock. Depending on reservoir permeability, fracture surface area, pumping pressure, etc., the flow rate varies significantly from project to project.

Along with the ongoing debate over the definition of EGS, it has also been reported that the output of EGS projects is far lower than the theoretical expectation. Sanyal and Butler (2005) built a number of simulation models as a starting point for estimating EGS reserves on the basis of conditions seen at a desert park in USA, which 
suggested a recovery factor greater than $40 \%$ for EGS. However, Grant and Garg (2012) later pointed out that the recovery factor for the Cooper Basin EGS system would be lower than $2 \%$, according to the modelled performance.

\section{Conclusions}

Many publications provide eye-catching numbers about EGS potential, yet there is still much to do to tap this energy. However, from this review of EGS projects worldwide, it transpires that EGS is still on a learning curve. Success is not guaranteed, and this implies significant financial risks for any EGS project, which can lead to its abandonment in some cases (e.g. Bad Urach project).

This observation leads to the natural question of why success is not guaranteed. From the classification exercise performed in this work, it is possible to conclude that the 'typical' EGS system does not exist, so much that, as shown in the introduction, the geothermal community does not even have a universally accepted and unambiguous definition of EGS as yet.

The typical EGS system does not exist because - as shown in the tables and in the figures - there are several possible (and significantly different) geological, petrophysical, thermal, hydraulic and geomechanical environments where high temperature can be tapped underground. Even the depth where sufficiently high temperature can be encountered varies from region to region in the world, making it difficult to specify what 'deep geothermal energy' (another term often used within the geothermal community) really is and how it can be related to the EGS concept.

The problem is that of handling each particular EGS system in such a way that economic flow rates at the right temperature and over a sufficient time span can be obtained. It is commonly accepted that for an EGS doublet system to be of commercial size, assuming a depth greater than $3 \mathrm{~km}$ and a temperature greater than $150^{\circ} \mathrm{C}$, the system should operate at flow rates between 50 and $100 \mathrm{l} / \mathrm{s}$ and produce an electric power of 3 to $10 \mathrm{MW}_{\mathrm{e}}$ over a life of at least 25 years (Jung 2013).

Based on the relatively limited EGS experience gathered to date and the extreme variety of natural occurrences and engineering solutions (including reservoir enhancement), it is therefore no surprise that EGS is still on a learning curve. This learning process must continue via more research and development, further technology advances and significantly more financial and political incentives before EGS will be commercially feasible, say in the next 10 to 20 years.

It is critical for EGS to ensure that relevant technologies are applied, having minimal risk of seismicity, and permitting the exploration of geothermal resource in a safe and environmentally friendly manner.

In the same vein, communities should be provided with regular, understandable and realistic information about EGS activities in order to gain public acceptance. Ongoing dialogue and interaction with communities are vital to achieve this. 
Received: 18 June 2013 Accepted: 30 August 2013

Published: 5 November 2013

\section{References}

AGRCC (Australian Geothermal Reporting Code Committee) (2010) Geothermal lexicon for resources and reserves definition and reporting, 2nd edn. Australian Geothermal Reporting Code Committee, Adelaide

AltaRock Energy Inc (2012) AltaRock EGS demonstration project status with NCPA at the Geysers., http://altarockenergy. com/AltaRock_EGS_Demonstration_Project_Status_101909.pdf. Accessed 19 Sept 2012

Battye DL, Ashman PJ (2009) Radiation associated with Hot Rock geothermal power. In: Budd AR, Gurgenci H (eds) Proceedings of the 2009 Australian Geothermal Energy Conference, Geoscience Australia, Record 2009/35. Commonwealth of Australia, Adelaide, 17-19 November 2010

Baumgärtner J (2012) Insheim and Landau - recent experiences with EGS technology in the Upper Rhine Graben. Oral presentation presented at ICEGS 2012, Freiburg, 25 May 2012

Bayerisches Landesamt für Wasserwirtschaft (2011) City of Altheim - Geothermal energy supply., http://ebookbrowse. com/27-slides-0-2-altheim-pdf-pdf-d183736900. Accessed 1 Nov 2012

Bertini G, Casini M, Gianelli G, Pandeli E (2006) Geological structure of a long-living geothermal system, Lardarello, Italy. Terra Nova 18:163-169

BGR (2012a) GeneSys Horstberg., http://www.genesys-hannover.de/Genesys/EN/Horstberg/horstberg_node_en.html Accessed 17 Sept. 2013

BGR (2012b) Milestones of the Genesys project., http://www.bgr.bund.de/Genesys/EN/Meilensteine/ meilensteine_inhalt_en.html. Accessed 24 Sept 2012

BGR (2013) GeneSys Project Aktuelles., http://www.genesys-hannover.de/Genesys/DE/Aktuelles/aktuelles_node.html Accessed 12 Jan 2013

Bine 4 (2012) Korrosion und Materialqualifizierung, http://www.bine.info/service/bestellen/download-print/publikation/ korrosion-in-geothermischen-anlagen/korrosion-und-materialqualifizierung/. Accessed 18 Sept. 2013

BINE (2012a) Korrosion in geothermischen Anlagen., http://www.bine.info/service/bestellen/download-print/publikation/ korrosion-in-geothermischen-anlagen/korrosion-und-materialqualifizierung/. Accessed 18 Sept. 2013

BINE (2012b) Insheim - Geothermieanlage mit neuem Konzept., http://www.bine.info/newsuebersicht/news/insheimgeothermieanlage-mit-neuem-konzept. Accessed 05 Sept 2012

BINE (2012c) Geothermische Stromerzeugung im Verbund mit Wärmenetz., http://www.bine.info/service/bestellen/ download-print/publikation/geothermische-stromerzeugung-im-verbund-mit-waermenetz/Accessed 21 Sept 2012

BINE (2012d) Projektinfo 14/07: Geothermische Stromerzeugung in Landau., http://www.bine.info/hauptnavigation/ publikationen/publikation/geothermische-stromerzeugung-in-landau/. Accessed 20 Sept 2012

BLM (Bureau of Land Management) (2012) Newberry geothermal exploration project., http://www.blm.gov/or/districts/ prineville/plans/newberry/index.php. Accessed 20 Sept 2012

Bloomquist R (2012) Integrating small power plants into agricultural projects., pangea.stanford.edu/ERE/pdf/ IGAstandard/EGC/szeged/I-8-01.pdf. Accessed 01 Nov 2012

Blöscher G, Zimmermann G, Moeck I, Huenges E (2012) Groß-Schönebeck (D) - the development of the learning curve: experience from the projects of recent years. Oral presentation presented at ICEGS 2012, Freiburg, 25 May 2012

BMU (2011) Tiefe Geothermie - Nutzungsmöglichkeiten in Deutschland, Beltz Bad Langensalza GmbH, BT Weimar

Bommer JJ, Oates S, Cepeda JM, Lindholm C, Bird J, Torres R, Marroquín G, Rivas J (2006) Control of hazard due to seismicity induced by a hot fractured rock geothermal project. Engineering Geology 83:287-306

Bracke R (2012) Geothermal energy - low enthalpy technologies. Oral presentation presented at Congreso Nacional de Energia 2012. CICR, San Jose/Costa Rica, 15-16 Feb 2012

Bromley C (2012) Geothermal induced seismicity: summary of international experience. Oral presentation presented at IEA-GIA Environmental Mitigation Workshop 2012, Taupo, 15-16 June 2012

Bromley CJ, Mongillo MA (2008) Geothermal energy from fractured reservoirs - dealing with induced seismicity. In: IEA OPEN Energy Technology Bulletin, Issue No. 48. IEA., http://www.iea.org/impagr/cip/pdf/Issue48Geothermal.pdf. Accessed 31 May 2013

Brown D (1995) The US Hot Dry Rock program - 20 years of experience in reservoir testing. Paper presented at world geothermal congress 1995, Firenze, 18-31 May 1995

Brown D (1997) Review of Fenton Hill HDR test results. Paper presented at New Energy and Industrial Technology Development Organization (NEDO) geothermal and HRD conference 1997, Sendai, 10-17 Mar 1997

Brown D (2009) Hot Dry Rock Geothermal Energy: important lessons from Fenton Hill. Paper presented at thirty-fourth workshop on geothermal reservoir engineering, Stanford University, Stanford, 9-11 Feb 2009

Cappetti G (2006) How EGS is investigated in the case of the Lardarello geothermal field. In: Conference abstracts of engine launching conference. BRGM, Orleans

Chabora E, Zemach E (2013) Desert Peak EGS Project. Geothermal Technologies office 2013 Peer Review, Presentation hold at the April 2013 peer review meeting in Denver, Colorado

Chabora E, Zemach E, Spielman P, Drakos P, Hickman S, Lutz S, Boyle K, Falconer A, Robertson-Tait A, Davatzes NC, Rose P, Majer E, Jarpe S (2012) Hydraulic stimulation of well 27-15, Desert Peak geothermal field, Nevada, USA. In: Proceedings of thirty-seventh workshop on geothermal reservoir engineering, Stanford University, Stanford, 30 Jan-1 Feb 2012

Cherubini Y, Cacace M, Scheck-Wenderoth M, Moeck I, Lewerenz B (2013) Controls on the deep thermal field: implications from 3-D numerical simulations for the geothermal research site Groß Schönebeck. Environ Earth Sci Special. doi:10.1007/s12665-013-2519-4

Cladouhos TT, Osborn WL, Petty S, Bour D, lovenitti J, Callahan O, Nordin Y, Perry D, Stern PL (2012) Newberry volcano EGS demonstration - phase I results. In: Proceedings of thirty-seventh workshop on geothermal reservoir engineering. , Stanford University, Stanford, 30 Jan-1 Feb 2012

Clauser C (2006) Geothermal energy. In: Heinloth K (ed) Landolt-Börnstein group VIII: advanced materials and technologies, vol. 3: energy technologies, subvol. C: renewable energies. Springer, Heidelberg 
Cornet FH (2012) The learning curve: the Le Mayet de Montagne experiment (1978-1987). Oral presentation presented at ICEGS 2012, Freiburg, 25 May 2012

Cotler S (2009) Enhanced geothermal energy project halted in the Geysers., http://stevecotler.com/tales/2009/09/ 03/egs-geysers-halted. Accessed 26 Sept 2012

Cummings RG, Morris GE (1979) Economic modeling of electricity production from Hot Dry Rock geothermal reservoirs: methodology and analysis. EA-630, Research Project 1017 LASL (LA-7888-HDR). OSTI Information Bridge., http://www.osti.gov/bridge/servlets/purl/5716131-wg4gUV/native/5716131.pdf. Accessed 31 May 2013

Davatzes N, Hickmann S, Zemach E, Spielman P, Robertson-Tait A, Drakos P, Lutz S, Rose P, Moore J, Majer E, Kennedy M, Stacey R, Swyer M (2012) Structural and geomechanical constraints in designing an EGS: example at Desert Peak Geothermal Field, Nevada. Oral presentation presented at ICEGS 2012, Freiburg, 25 May 2012

DiPippo R (2012a) Geothermal power plants, 3rd edn. Elsevier, New York, pp 451-456

DiPippo R (2012b) Geothermal power plants, 3rd edn. Elsevier, New York, pp 463-474

Duchane D (1998) The history of HDR research and development. In: Draft proceedings of the 4th international HDR forum, Strasbourg, 28-30 Sept 1998

Dumas P (2010) NER300: what for geothermal? Oral presentation held at second EGEC TP geoelec meeting, Brussels, 24 Mar 2010

EGEC (European Geothermal Energy Council) (2012) Geothermal market report, 2nd edition. European Geothermal Energy Council, Brussels, December 2012

EGEC (European Geothermal Energy Council) (2013) Fact sheet on enhanced geothermal systems: why it is different to shale gas. Accessed via the EGEC News, Issue No. 35., June 2013

EGS Coso (2013) Coso/EGS program., http://egs.egi.utah.edu/indexcoso.htm. Accessed 28 May 2013

Enbw (2013) Energie, die aus der Tiefe kommt - Das Geothermiekraftwerk in Bruchsal., http://www.enbw.com/ content/de/der_konzern/_media/pdf/Flyer_Geothermie-V4silber-neu-grafik.pdf. Accessed 30 Jan 2013

ENGINE (2008a) ENGINE - geothermal lighthouse projects in Europe - Lardarello., http://engine.brgm.fr/mediapages/ lighthouseProjects/LH-Quest_HydroTherm_2_Larderello.pdf. Accessed 17 Sept. 2013

ENGINE (2008b) ENGINE coordination action. Best practice handbook for the development of unconventional geothermal resources with a focus on enhanced geothermal system. BRGM, Orleans, http://engine.brgm.fr/ Documents/ENGINE_BestPracticeHandbook.pdf. Accessed 25 Jan 2013

ENGINE (2012) ENGINE- geothermal lighthouse projects in Europe., http://engine.brgm.fr/mediapages/ lighthouseProjects/LH-Quest_EGS_4_GeneSys.pdf. Accessed 24 Sept 2012

Evans K (2011) Enhanced/engineered geothermal systems: - experiences to date and lessons learned. In: Abstract volume of 9th Swiss geoscience meeting. ETH Zürich, Zürich, 11-13 Nov 2011

Exorka (2013) F\&E-Projekt Geothermie Allgäu 2.0., http://cif-ev.de/pdf/block2/P1.pdf Accessed 22 May 2013

Feige S, Roloff R (2012) Geothermal energy production - a subject for radiation protection. Paper presented at EUROSAFE Forum 2012, Brussels, 5-6 Nov 2012

Feldbusch E, Regenspurg S, Banks J, Milsch H, Saadat A (2013) Alteration of fluid properties during the initial operation of a geothermal plant: results from in situ measurements in Groß Schönebeck. Environ Earth Sci. doi:10.1007/ s12665-013-2409-9

Fittermann DV (1988) Overview of the structure and geothermal potential of Newberry Volcano, Oregon. J Geophys Res 93:10059-10066

Garcia J, Walters M, Beall J, Hartline C, Pingol A, Pistone S, Wright M (2012) Overview of the Northwest Geysers EGS Demonstration Project. In: Proceedings of the thirty-seventh workshop on geothermal reservoir engineering (ed) Proceedings of the thirty-seventh workshop on geothermal reservoir engineering. Stanford University, Stanford, 30 Jan-1 Feb 2012

Gebo NDS (Forschungsverbund Geothermie und Hochleistungsbohrtechnik) (2012a) Current state of research., http:// www.gebo-nds.de/en/research-project/current-state-of-research/. Accessed 12 Sept 2012

Gebo NDS (Forschungsverbund Geothermie und Hochleistungsbohrtechnik) (2012b) Research project., http://www. gebo-nds.de/en/research-project/. Accessed 20 Sept 2012

GeneSys (2012) GeneSys Hannover., http://www.genesys-hannover.de/Genesys/DE/Hannover/hannover_node.html. Accessed 3 June 2013

Genter A (2012) Lessons learned from projects in the early stage of EGS development: Soultz-sous-Fôrets (F). Oral presentation held at ICEGS 2012, Freiburg, 25 May 2012

Geodynamics (2013) Innamincka Deeps (EGS) project., http://www.geodynamics.com.au/Our-Projects/InnaminckaDeeps.aspx. Accessed: 18 Sept. 2013

Geothermie Stadt St. Gallen (2013a) Das Geothermie-Projekt der Stadt St. Gallen., http://www.geothermie.stadt.sg.ch/ projekt.html. Accessed 21 May 2013

Geothermie Stadt St. Gallen (2013b) Das Geothermie-Projekt geht weiter., http://www.geothermie.stadt.sg.ch/aktuell/ details/artikel/das-geothermie-projekt-geht-weiter.html. Accessed 29 August 2013

Geothermie-Nachrichten (2012) Pfalz: Geothermiekraftwerk in Insheim geht ans Netz., http://www.geothermienachrichten.de/pfalz-geothermiekraftwerk-in-insheim-geht-ans-netz. Accessed 16 Nov 2012

Geothermie-Pfalz (2013) Geothermieprojekt Insheim., http://geothermie-pfalz.de/geoinsh.html. Accessed 26 Aug 2013

Giardini D (2009) Geothermal quake risks must be faced. Nature 462:848-849

Goldstein B, Hiriart G, Bertani R, Bromley C, Gutiérrez-Negrín L, Huenges E, Muraoka H, Ragnarsson A, Tester J, Zui V (2011) Geothermal Energy. In: Edenhofer O, Pichs-Madruga R, Sokona Y, Seyboth K, Matschoss P, Kadner S, Zwickel T, Eickemeier P, Hansen G, Schlomer S, von Stechow C (eds) IPCC special report on renewable energy sources and climate change mitigation. Cambridge University Press, Cambridge, p 406

Grant MA, Garg SK (2012) Recovery factor for EGS. In: Proceedings of the 37th workshop on geothermal reservoir engineering. Stanford University, Stanford, 30 Jan-1 Feb 2012

Groos JC, Grund M, Ritter JRR (2012) Automated detection of microseismic events in the Upper Rhine valley near the city of Landau/South Palatinate. Geophys Res Abstracts 14:EGU2012-10482, 2012, EGU General Assembly 2012 
GtV (Bundesverband Geothermie) (2013) Liste der tiefen Geothermieprojekte in Deutschland. Liste der tiefen Geothermieprojekte in Deutschland, Liste der tiefen Geothermieprojekte in Deutschland, http://www. geothermie.de/wissenswelt/geothermie/in-deutschland.html. Accessed 20 Aug 2013

Häring MO (2007) Geothermische Stromproduktion aus Enhanced Geothermal Systems (EGS) Stand der Technik., www. geothermal.ch/fileadmin/docs/downloads/egs061207.pdf. Accessed 30 Jan 2013

Häring MO, Hopkirk R (2002) The Swiss deep heat mining project - the Basel exploration drilling. GHC Bulletin 23(1):31-33

Henninges J, Brandt W, Erbas K, Moeck I, Saadat A, Reinsch T, Zimmermann G (2012) Downhole monitoring during hydraulic experiments at the in-situ geothermal lab Groß Schönebeck. In: Proceedings of the thirty-seventh workshop on geothermal reservoir engineering. Stanford University, Stanford, 30 Jan 1-Feb 2012

Holl H (2012) Geodynamics Update: Innamincka Deeps EGS Project. Oral presentation held at ICEGS 2012, Freiburg, 25 May 2012

Huenges E (2010) Geothermal energy systems - exploration, development, and utilization. Wiley, Weinheim

Huenges E (2013) Personal communication on 18th of January

Ilyasov M, Ostermann I, Punzi A (2010) Modeling deep geothermal reservoirs: recent advances and future problems. In: Freeden W, Nashed MZ, Sonar T (eds) Handbook of geomathematics, vol. 1: general issues, key technologies, data acquisition, modeling the system earth. Springer, Berlin, pp 689-694

Informationsportal Tiefe Geothermie (2012) Beginn der Stimulation im EGS-Projekt Newberry Geothermal., http://www. tiefegeothermie.de/top-themen/beginn-der-stimulation-im-egs-projekt-newbern-geothermal. Accessed 21 May 2013

Informationsportal Tiefe Geothermie (2013a) Mauerstetten soll als Forschungsprojekt neu erschlossen werden., http://www. tiefegeothermie.de/news/mauerstetten-soll-als-forschungsprojekt-neu-erschlossen-werden. Accessed 21 May 2013

Informationsportal Tiefe Geothermie (2013b) Arbeitsmedien zur Stromerzeugung in Geothermiekraftwerken., http://www. tiefegeothermie.de/top-themen/arbeitsmedien-zur-stromerzeugung-in-geothermiekraftwerken Accessed 21 May 2013

Insheim (2012) Insheim., http://www.insheim.de/wirtschaft/geothermie.html. Accessed 9 May 2012

Johansson TB, Kelly H, Reddy AK, Williams RH (eds) (1993) Renewable energy: sources for fuels and electricity, 2nd edn. Island Press, Washington D.C

Julian BR, Foulger GR, Monastero FC (2009) Seismic monitoring of EGS stimulation tests at the Coso Geothermal Field, California, using microearthquake locations and moment tensors. In: Proceedings of the thirty-fourth workshop on geothermal reservoir engineering. Stanford University, Stanford

Jung R (2013) EGS-goodbye or back to the future, effective and sustainable hydraulic fracturing. In: Jeffrey R (ed) InTech.. doi:10.5772/56458, http://www.intechopen.com/books/effective-and-sustainable-hydraulic-fracturing/egsgoodbye-or-back-to-the-future-95. ISBN 978-953-51-1137-5. Accessed: 18 Sept 2013

Jupe AJ, Green ASP, Wallroth T (1992) Induced microseismicity and reservoir growth at the Fjällbacka hot dry rocks project, Sweden. Int J Rock Mechanics Mining Sci Geomechanics Abstracts 29(4):343-354

Kaieda H, Ito H, Kiho K, Suzuki K, Suenaga H, Shin K (2005) Review of the Ogachi HDR project in Japan. In: Proceedings of the world geothermal congress 2005, Antalya, 24-29 April 2005

Kaieda H, Sasaki S, Wyborn D (2010) Comparison of characteristics of micro-earthquakes observed during hydraulic stimulation operations in Ogachi, Hijiori and Cooper Basin HDR projects. In: Proceedings of the World Geothermal Congress 2010, Bali, 25-29 April 2010

Kappelmeyer O, Jung R (1987) HDR experiments at Falkenberg/Bavaria. Geothermics 16:375-392

Karner SL (2005) Stimulation techniques used in enhanced geothermal systems: perspectives from geomechanics and rock physics. In: Proceedings of the thirtieth workshop on geothermal reservoir engineering. Stanford University, Stanford, 31 Jan-2 Feb 2005

KIT (2013) Langzeitbetrieb und Optimierung eines Geothermiekraftwerks in einem geklüftet-porösen Reservoir im Oberrheingraben (LOGRO)., http://www.agw.kit.edu/908 998.php Accessed 20 Sept 2012

Kreuter H (2011) Deep geothermal projects in Germany - status and future development., http://www.renewablesb2b. com/data/shared/GEO_PPT_Kreuter.pdf. Accessed 28 Sept 2012

Lacirignola M, Blanc I (2012) Environmental analysis of practical design options for enhanced geothermal systems (EGS) through life-cycle assessment. Renew Energy 50:901-914

Ladner F, Häring MO (2009) Hydraulic characteristics of the basel 1 enhanced geothermal system. GRC Transactions 33:199-203

Lazzarotto A, Sabatelli F (2005) Technological developments in deep drilling in the Lardarello area. In: Proceedings of the world geothermal congress 2005, Antalya, 24-29 April 2005

LGB-rlp (2012) (Landesamt für Geologie und Bergbau Rheinland-Pfalz) Steckbrief: Projektstandort Insheim., http://www. lgb-rlp.de/projektstandort_insheim.html. Accessed 06 Sept 2012

Lutz SJ, Schriener A Jr, Schochet D, Robertson-Tait A (2003) Geologic characterization of pre-tertiary rocks at the Desert Peak East EGS project site, Churchill County, Nevada. Geoth Res T 27:865-870, 12-15 Oct 2003

Majer E, Baria R, Stark M, Oates S, Bommer J, Smith B, Asanuma H (2007) Induced seismicity associated with enhanced geothermal systems. Geothermics 36:185-222

Majer E, Nelson J, Robertson-Tait A, Savy J, Wong I (2011) Protocol for addressing induced seismicity associated with enhanced geothermal systems., http://www1.eere.energy.gov/geothermal/pdfs/ geothermal_seismicity_protocol_012012.pdf. Accessed 05 June 2013

Majer E, Nelson J, Robertson-Tait A, Savy J, Wong I (2013) Best practices for addressing induced seismicity associated with enhanced geothermal systems (EGS), DRAFT, 23 May 2013., http://esd.lbl.gov/files/research/projects/ induced_seismicity/egs/Best_Practices_EGS_Induced_Seismicity_Draft_May_23_2013.pdf. Accessed 29 Aug 2013

MIT, Tester JW, Anderson BJ, Batchelor AS, Blackwell DD, DiPippo R, Drake EM, Garnish J, Livesay B, Moore MC, Nichols K, Petty S, Toksöz MN, Veatch RW Jr (2006a) The Future of geothermal energy - impact of enhanced geothermal systems on the United States in the 21st Century. US Department of Energy, Washington, D.C., pp 1-10

MIT, Tester JW, Anderson BJ, Batchelor AS, Blackwell DD, DiPippo R, Drake EM, Garnish J, Livesay B, Moore MC, Nichols K, Petty S, Toksöz MN, Veatch RW Jr (2006b) The future of geothermal energy - impact of enhanced geothermal systems on the United States in the 21st Century. US Department of Energy, Washington, D.C., pp 4-40

MIT, Tester JW, Anderson BJ, Batchelor AS, Blackwell DD, DiPippo R, Drake EM, Garnish J, Livesay B, Moore MC, Nichols K, Petty S, Toksöz MN, Veatch RW Jr (2006c) The future of geothermal energy - impact of enhanced geothermal systems on the United States in the 21st Century. US Department of Energy, Washington, D.C., p 4 
MIT, Tester JW, Anderson BJ, Batchelor AS, Blackwell DD, DiPippo R, Drake EM, Garnish J, Livesay B, Moore MC, Nichols K, Petty S, Toksöz MN, Veatch RW Jr (2006d) The future of geothermal energy - impact of enhanced geothermal systems on the United States in the 21st Century. US Department of Energy, Washington, D.C., pp 4-26

MIT, Tester JW, Anderson BJ, Batchelor AS, Blackwell DD, DiPippo R, Drake EM, Garnish J, Livesay B, Moore MC, Nichols K, Petty S, Toksöz MN, Veatch RW Jr (2006e) The future of geothermal energy - impact of enhanced geothermal systems on the United States in the 21st Century. US Department of Energy, Washington, D.C., pp 4-37

MIT, Tester JW, Anderson BJ, Batchelor AS, Blackwell DD, DiPippo R, Drake EM, Garnish J, Livesay B, Moore MC, Nichols K, Petty S, Toksöz MN, Veatch RW Jr (2006f) The future of geothermal energy - impact of enhanced geotherma systems on the United States in the 21st Century. US Department of Energy, Washington, D.C., pp 4-14, 4-18

MIT, Tester JW, Anderson BJ, Batchelor AS, Blackwell DD, DiPippo R, Drake EM, Garnish J, Livesay B, Moore MC, Nichols K, Petty S, Toksöz MN, Veatch RW Jr (2006g) The future of geothermal energy - impact of enhanced geothermal systems on the United States in the 21st Century. US Department of Energy, Washington, D.C., p 4-7-4-13

MIT, Tester JW, Anderson BJ, Batchelor AS, Blackwell DD, DiPippo R, Drake EM, Garnish J, Livesay B, Moore MC, Nichols K, Petty S, Toksöz MN, Veatch RW Jr (2006h) The future of geothermal energy - impact of enhanced geothermal systems on the United States in the 21st Century. US Department of Energy, Washington, D.C., pp 4-38

MIT, Tester JW, Anderson BJ, Batchelor AS, Blackwell DD, DiPippo R, Drake EM, Garnish J, Livesay B, Moore MC, Nichols K, Petty S, Toksöz MN, Veatch RW Jr (2006i) The future of geothermal energy - impact of enhanced geothermal systems on the United States in the 21st Century. US Department of Energy, Washington, D.C., p 4

Noack V, Scheck-Wenderoth M, Cacace M, Schneider M (2013) Influence of fluid flow on the regional thermal field: results from 3D numerical modelling for the area of Brandenburg (North German Basin). Environ Earth Sci. doi:10.1007/s12665-013-2438-4

Parker R (1999) The Rosemanowes HDR project 1983-1991. Geothermics 28:603-615

Pernecker G (1999) Altheim geothermal plant for electricity production by ORC-turbogenerator. In: Peter L (ed) Bulletin d'hydrogéologie No 17. Centre d'Hydrogéologie. Université de Neuchâtel, Altheim, Austria

Petratherm (2012) Paralana., http://www.petratherm.com.au/projects/paralana. Accessed 19 Sept 2012

Pfalzwerke-geofuture (2012) Projekt Insheim., http://www.pfalzwerke-geofuture.de/6240.php. Accessed 05 Sept 2012

Pierce V (2011) Introduction to geothermal power. The English Press, Delhi

Portier S, André L, Vuataz FD (2007) Review on chemical stimulation techniques in oil industry and applications to geothermal systems. Technical report in enhanced geothermal innovative network for Europe. CREGE - Centre for Geothermal Research, Neuchâtel

Potter R, Robinson E, Smith M (1974) Method of extracting heat from dry geothermal reservoirs. US Patent No. 3,786,858, USA. Los Alamos, New Mexico. Accessed: 18 Sept 2013

Prevost JKJ (2004) The geothermal energy industry of El Salvador. Term Paper - ESD.166J Sustainable Energy, Spring 2004., web.mit.edu/10.391J/www/proceedings/Geothermal_Prevost2004.pdf. Accessed 26 Sept 2012

Regenspurg S, Blöcher G (2012) Saadat A (2012) Impact of geothermal stimulation treatment on the environment - a risk assessment. In: International conference on enhanced geothermal systems, Freiburg

Rettenmaier D (2012) Lessons Learned - Reservoirmanagement Bruchsal., http://www.ta-survey.nl/pdf/GU2012Detlev_Rettenmaier.pdf. Accessed 06 June 2013

Richards HG, Savage D, Andrews JN (1992) Granite-water reactions in an experimental hot dry rock geothermal reservoir, Rosemanowes test site, Cornwall, U.K. Appl Geochem 7(3):193-222

Rodríguez JA (2000) Geothermal development in El Salvador - a country update. In: Proceedings of the world geothermal congress 2000. Kyushu - Tohoku, 28 May-10 Jun 2000

Rodríguez JA (2003) Geothermal El Salvador., www.geothermal.org/articles/elsalvador.pdf. Accessed 26 Sept 2012

Rodríguez JA (2008) El Salvador Geothermal., http://www.un.org/esa/sustdev/sids/2008_roundtable/presentation/ energy_rodriguez.pdf. Accessed 26 Sept 2012

Romano B (2009) Geologist on trial in Basel: two EGS projects shuttered., http://www.rechargenews.com/energy/ geothermal/article202104.ece. Accessed 26 Sept 2012

Romero A Jr, McEvilly TV, Majer E, Vasco D (1995) Characterization of the geothermal system beneath the Northwest Geysers Steam Field, California, from seismicity and velocity patterns. Geothermics 24:471-487

Rose P, Moore J, Kovac K, Adams M, McCulloch J, Spielman P, Sheridan J, Hickman S, Davatzes N, Julian B, Foulger G, Weidler R (2004) The Coso EGS Project - recent developments. Paper presented at Great Basin Geothermal Workshop, Reno, 5 Nov 2004

Sanjuan B, Jousset P, Pajot G, Debeglia N, De Michele M, Brach M, Dupont F, Braibant G, Lasne E, Duré F (2010) Monitoring of Bouillante geothermal exploitation (Guadeloupe, French West Indies) and the impact on its immediate environment. In: Proceedings of the world geothermal congress 2010, Bali, 25-30 April 2010

Sanyal SK, Butler SJ (2005) An analysis of power generation prospects from enhanced geothermal systems. In: Proceedings of the world geothermal congress 2005, Antalya, 24-29 April 2005

Sasaki S (1998) Characteristics of microseismic events induced during hydraulic fracturing experiments at the Hijiori hot dry rock geothermal energy site, Yamagata, Japan. Tectonophysics 289:171-188

Schanz U, Stang H, Tenzer H, Homeier G, Hase M, Baisch S, Weidler R, Macek A, Uhlig S (2003) Hot dry rock project Urach - a general overview. In: Proceedings of the European geothermal conference, Szeged, 25-30 May 2003

Schrage C, Bems C, Kreuter H, Hild S, Volland S (2012a) Overview of the enhanced geothermal energy project in Mauerstetten, Germany., http://www.ta-survey.nl/index.php?id=109\&lang=EN. Accessed 07 Sept 2012

Schrage C, Bems C, Kreuter H, Hild S, Volland S (2012b) Geothermie Allgäu 2.0 - overview of the enhanced geothermal energy project in Mauerstetten. Oral presentation held at Amsterdam, Germany, 18 Apr 2012

Schulte T, Zimmermann G, Vuataz F, Portier S, Tischner T, Junker R, Jatho R, Huenges E (2010) Enhancing geothermal reservoirs. In: Huenges E (ed) Geothermal energy systems. Wiley, Weinheim

Stephens JC, Jiusto S (2010) Assessing innovation in emerging energy technologies: socio-technical dynamics of carbon capture and storage (CCS) and enhanced geothermal systems (EGS) in the USA. Energy Policy 38:2020-2031

Tenzer H (2001) Development of hot dry rock technology. GHC Bulletin, December, 2001 
Tenzer H, Schanz U, Homeier G (2000) HDR research programme and results of drill hole Urach 3 to depth of $4440 \mathrm{~m}$ the key for realisation of a HDR programme in Southern Germany and Northern Switzerland. In: Proceedings of the world geothermal congress. Kyushu, Tohoku, 25-30 April 2010

Tester JW, Brown DW, Potter RM (1989) Hot dry rock geothermal energy - a new energy agenda for the 21st century. Los Alamos National Laboratory report LA-11514-MS. US Department of Energy, Washington D.C.

Tischner T, Evers H, Hauswirth H, Jatho R, Kosinowski M, Sulzbacher H (2010) New concepts for extracting geothermal energy from one well: the GeneSys-Project. In: Proceedings of the world geothermal congress, Bali, 25-30 April 2010

Wallroth T, Eliasson T, Sundquist U (1999) Hot dry rock research experiments at Fjällbacka, Sweden. Geothermics 28:617-625

Walters M (2013) Demonstration of an Enhanced Geothermal System at the Northwest Geysers Geothermal Field, CA Geothermal Technologies Office 2013 Peer Review, Presentation hold at the April 2013 peer review meeting in Denver, Colorado

Williams CF, Reed MJ, Anderson AF (2011) Updating the classification of geothermal resources. In: Proceedings of the thirty-sixth workshop on geothermal reservoir engineering. Stanford University, Stanford, 31 Jan-2 Feb 2011

Wyborn D (2011) Hydraulic stimulation of the Habanero enhanced geothermal system (EGS), South Australia. Presentation held at the 5th BC unconventional gas technical forum., April 2011 http://datafind.gov.bc.ca/query. html?ap $=\&$ style=ener\&qt=mayet\&Submit.x=0\&Submit.y=0 Accessed 10 Sept 2012

Wyborn D, Graaf L, Davidson S, Hann S (2005) Development of Australia's first hot fractured rock (HFR) underground heat exchanger, Cooper Basin, South Australia. In: Proceedings of the world geothermal congress, Antalya, 24-29 April 2005

Zimmermann G, Tischner T, Legarth B, Huenges E (2009) Pressure-dependent production efficiency of an enhanced geothermal system (EGS): stimulation results and implications for hydraulic fracture treatments. Pure Appl Geophys 166:1089-1106

doi:10.1186/2195-9706-1-4

Cite this article as: Breede et al: A systematic review of enhanced (or engineered) geothermal systems: past, present and future. Geothermal Energy 2013 1:4.

\section{Submit your manuscript to a SpringerOpen ${ }^{\circ}$ journal and benefit from:}

- Convenient online submission

- Rigorous peer review

- Immediate publication on acceptance

- Open access: articles freely available online

- High visibility within the field

- Retaining the copyright to your article

Submit your next manuscript at $\gg$ springeropen.com 